\title{
Output control of continuous-time uncertain switched linear systems via switched static output feedback
}

\author{
Leonardo Ataide Carniato, Alexandre Ataide Carniato, Marcelo Carvalho \\ Minhoto Teixeira, Rodrigo Cardim, Edson Italo Mainardi Junior \& Edvaldo \\ Assunção
}

To cite this article: Leonardo Ataide Carniato, Alexandre Ataide Carniato, Marcelo Carvalho Minhoto Teixeira, Rodrigo Cardim, Edson Italo Mainardi Junior \& Edvaldo Assunção (2018): Output control of continuous-time uncertain switched linear systems via switched static output feedback, International Journal of Control, DOI: 10.1080/00207179.2018.1495341

To link to this article: https://doi.org/10.1080/00207179.2018.1495341

Accepted author version posted online: 02

Jul 2018.

Published online: 17 Jul 2018.

Submit your article to this journal $₫$

ЦIl Article views: 130

View Crossmark data \lceil 


\title{
Output control of continuous-time uncertain switched linear systems via switched static output feedback
}

\author{
Leonardo Ataide Carniato (ib) ${ }^{\mathrm{a}, \mathrm{b}}$, Alexandre Ataide Carniato (1)a, Marcelo Carvalho Minhoto Teixeira $\mathbb{1}^{\mathrm{b}}$, Rodrigo \\ Cardim $\mathbb{1}^{\mathrm{b}}$, Edson Italo Mainardi Junior (10c and Edvaldo Assunção (10) \\ ${ }^{\text {a}}$ Federal Institute of São Paulo, Campus Presidente Epitácio, Presidente Epitácio, Brazil; b School of Natural Sciences and Engineering, São Paulo State \\ University (UNESP), Ilha Solteira, Brazil; ${ }^{C}$ Federal Institute of Mato Grosso do Sul, Campus Três Lagoas, Três Lagoas, Brazil
}

\begin{abstract}
This paper is devoted to the robust $\mathscr{H}_{\infty}$ and guaranteed cost control problems for continuous-time switched linear systems with polytopic uncertainties, considering an output-dependent switching law and a switched static output feedback controller. The proposed method offers new sufficient conditions based on Linear Matrix Inequalities (LMIs) for designing the switching strategy. In order to provide extra free dimensions in the solution space, some conditions become a special class of Bilinear Matrix Inequalities (BMIs). Therefore, the hybrid algorithm Differential Evolution-Linear Matrix Inequality is proposed for obtaining feasible solutions of this NP-hard problem. Theoretical analyses and numerical examples show that these new procedures reduce the design conservatism of two recent known methods for solving the presented control problems. Besides, a practical application of the method in the design and simulation of a robust switched controller of a switched semi-active suspension is performed.
\end{abstract}

\section{ARTICLE HISTORY}

Received 17 November 2017

Accepted 20 June 2018

\section{KEYWORDS}

Robust output switching strategy; switched static output feedback; robust control; linear matrix inequalities; robust static output feedback $\mathscr{H}_{\infty}$ control

\section{Introduction}

Recently, the designing of control laws for switched systems have received lots of attention ( $\mathrm{Yu} \& \mathrm{Wu}, 2015$; Zhang, Zhuang, \& Braatz, 2016). Switched systems are a particular case of hybrid systems which are composed of a family of subsystems where a switching rule or strategy defines the active subsystem at each instant of time (Liberzon, 2003). The growing interest in this topic is mainly due their widespread practical applications, such as power electronics (Cardim, Teixeira, Assunção, \& Covacic, 2009; Deaecto, Geromel, Garcia, \& Pomilio, 2010), embedded systems (Zhang \& Hu, 2008), road traffic control strategies (Papageorgiou, Diakaki, Dinopoulou, Kotsialos, \& Wang, 2003), among others. A significant result concerning the stability of switched linear systems was presented in Wicks, Peleties, and DeCarlo (1994): it was demonstrated that if there exists a Hurwitz convex combination of the subsystems matrices, then there exists a state switching rule that stabilises the switched linear system. Regarding the concepts of robust stabilisation, Zhai, Lin, and Antsaklis (2003) proposed a quadratic stabilisation rule for uncertain switched linear systems based on LMIs. In Lin and Antsaklis (2007) were developed two necessary and sufficient conditions for providing global stability for a class of switched linear systems with time-variant parametric uncertainties. Concerning stability and stabilisability of switched linear systems, in Lin and Antsaklis (2009) can be found a survey of available results and a proposed necessary and sufficient condition for asymptotic stabilisability.
Additionally, in Daafouz, Riedinger, and Iung (2002) the authors proposed two different LMI-based conditions. In the first one, it is presented a classical method while the second incorporates slack variables in order to relax the conditions. Moreover, in Ding and Yang (2009) the authors describe more relaxed conditions through Finsler's Lemma and piecewise quadratic Lyapunov functions for Static Output Feedback (SOF) control. For robust stabilisation of switched linear systems, when all subsystems matrices are not Hurwitz, in Yu and $\mathrm{Wu}$ (2015) are presented sufficient conditions, under some assumptions, for stability using the invariant subspace theory and average dwell time method. Considering some hypotheses, the authors in Yu and Zhao (2016) developed a necessary condition of stability for discrete-time switched linear systems. Concerning the output feedback control design problem for uncertain switched linear systems, its solution is among one of the most challenging problems in literature, due to their non-convex characteristic (Sadabadi \& Peaucelle, 2016; Syrmos, Abdallah, Dorato, \& Grigoriadis, 1997). Nevertheless, in recent years, the design of SOF controllers has been scrutinised by several authors, mainly due to the use of output feedback techniques results in simpler implementation routines for practical applications. In Peaucelle and Arzelier (2005), the authors proposed a two-step iterative algorithm focused on $\mathrm{H}_{2}$ optimisation. In doing so, Agulhari, Oliveira, and Peres (2010) presented an extension of previous method considering polynomial Lyapunov functions.

CONTACT Leonardo Ataide Carniato leonardo@ifsp.edu.br $\Theta$ School of Natural Sciences and Engineering, São Paulo State University (UNESP), Ilha Solteira, Av. José Carlos Rossi 1370, Ilha Solteira 15385-000, SP, Brazil 
Concerning a performance criterion, in this case, the $\mathscr{H}_{\infty}$ cost, several authors have proposed conditions LMI-based considering an output feedback strategy. Crusius and Trofino (1999) presented sufficient conditions for SOF controllers adopting linear matrices equalities. Aiming to avoid the equality constraints, Dong and Yang (2013) presented new LMI conditions, for cases where the output matrix is not required to be of full row rank. In sequence, the authors in Chang, Park, and Zhou (2015) have extended the flexibility of conditions for robust SOF $\mathscr{H}_{\infty}$ controller design. More specifically, the developed method is applicable for uncertain systems relaxing the constraint in system matrices. Furthermore, in Shi, Wang, Ren, and Fei (2017), the authors investigated the dynamic output feedback $\mathscr{H}_{\infty}$ control for a class of switched systems with mode-dependent average dwell time switching. In $\mathrm{Wu}, \mathrm{Gao}, \mathrm{Liu}$, and $\mathrm{Li}$ (2017), it was proposed a sliding mode control (SMC) for stochastic systems via output feedback considering among others, the exogenous disturbance constraint in SMC design. An extended state observer was used in order to reject external disturbance considering SMC for power converters (Liu, Vazquez, Wu, Marquez, Gao, \& Franquelo, 2017). Additionally, in Ban, Kwon, Won, and Kim (2018), it was designed a technique for robust $\mathscr{H}_{\infty}$ finite-time control for discrete-time polytopic uncertain switched linear systems.

In practical applications, the state vector may not be completely available. In this situation, it is important to aim at strategies for switching based on the measured output of the plant. Nowadays, to the best of the author's knowledge, there are not available in the literature papers which consider switched SOF $\mathscr{H}_{\infty}$ controllers design for uncertain switched linear systems with output-depending switching. Regarding the aforementioned researches, usually, papers on this subject consider full or reduced order output feedback controllers through of estimated state-dependent switching or state feedback.

The major contribution proposed in this paper is an exclusively output-dependent switching strategy jointly with the design of switched SOF $\mathscr{H}_{\infty}$ controllers. It is important to highlight that the proposed methodology also provides conditions to design switched output feedback $\mathscr{H}_{\infty}$ controllers for plants with only one dynamic subsystem. These two different situations are detailed in numerical examples.

Two different strategies to design output feedback controllers considering output dependent switching strategy for uncertain switched linear systems are presented. Firstly, it is considered that not exist exogenous input neither control input. In sequence, the results presented in Mainardi Júnior et al. (2015) are relaxed and the inclusion of guaranteed cost performance and decay rate criterion is approached. Following, novel conditions are proposed based on less conservative results available in Liu and Zhang (2003), Teixeira, Assunção, and Avellar (2003) and Mozelli and Palhares (2011).

Besides that, novel and less conservative conditions for switching SOF $\mathscr{H}_{\infty}$ control of continuous time switched linear systems are proposed. The results presented in Chang et al. (2015) are relaxed considering the inclusion of switched output feedback $\mathscr{H}_{\infty}$ controllers jointly with an output-dependent switching strategy.

In doing so, some bilinear terms appear in conditions of the proposed theorems. The conditions of the proposed methods are a special class of BMIs (Bilinear Matrix Inequalities), which contain some bilinear terms as the product of a matrix and a scalar, related to a suitable convex combination and two scalar parameters to provide extra free dimensions in the solution space. Currently, to the best of the authors' knowledge, there are not available solvers (deterministic methods) able to find the optimum solution for non-convex problems. Thus, the proposed design method of the output gains in order to stabilise an uncertain switched linear system is an NP-hard problem (Lin \& Antsaklis, 2009). Therefore, it is proposed the use of a hybrid metaheuristic technique, called DE-LMI (Differential Evolution - Linear Matrix Inequality) (Storn \& Price, 1997) for finding quasi-optimum values for SOF gains (Sandou, 2013) and/or a suitable convex combination. The proposed procedure can also be used for designing robust controllers for uncertain plants subject to structural failures, considering the plant uncertainties and the structural failures as polytopic uncertainties (Silva, Assunção, Teixeira, \& Cardim, 2013). An example illustrates a practical application of the method in the design and simulation of a robust controller of a semi-active suspension addressed in Cardim et al. (2016) and Geromel, Colaneri, and Bolzern (2008). The paper is organised as follows.

Section 2 presents a general definition of polytopic uncertain switched linear systems.

Following, in Section 3 the problem statement is given and relaxation results for robust SOF control design for uncertain switched linear systems with an output dependent switching law introduced in Mainardi Júnior et al. (2015) jointly with a performance criterion. Based on the relaxation concepts available in Liu and Zhang (2003), Teixeira et al. (2003) and Mozelli and Palhares (2011) are proposed novel conditions for stability of uncertain switched linear systems. A theoretical analysis shows that these conditions hold when the conditions presented in Mainardi Júnior et al. (2015) hold. A numerical example illustrates the flexibility obtained through of these less conservative conditions comparing feasible area and guaranteed cost obtained in the theorems proposed in this section.

In Section 4, it is presented the results for robust SOF $\mathscr{H}_{\infty}$ control (Chang et al., 2015). In the sequence, the problem statement is given and it is developed novel and less conservative conditions for robust switching SOF $\mathscr{H}_{\infty}$ control of continuous time switched linear systems. Furthermore, the results available in Chang et al. (2015) are generalised through a switched output $\mathscr{H}_{\infty}$ controller. A theoretical analysis shows that these new conditions hold when the conditions presented in Chang et al. (2015) hold. Finishing the contributions of this section, it is showed that the proposed methodology to develop robust SOF $\mathscr{H}_{\infty}$ switched controllers can be directly applied to nonswitched linear systems.

In Section 5, it is briefly described how the differential evolution (DE) is applied in order to solve the proposed control problem.

Three examples in Section 6 illustrate the effectiveness of the proposed methods, including the design and simulation results of a robust controller of a semi-active suspension (Cardim et al., 2016) and showing that there exist cases where the proposed conditions hold and the conditions from Mainardi Júnior et al. 
(2015) do not hold. Another example shows that the obtained $\mathscr{H}_{\infty}$ guaranteed cost from conditions proposed in this paper is less when compared to the cost achieved in Chang et al. (2015).

Finally, in Section 7, conclusions are presented and future works extensions are discussed.

The notation used in this paper are described as follows. For real matrices or vectors $(')$ indicates transpose. The set composed by the first $N$ positive integers $\{1, \ldots, N\}$ is represented by $\mathbb{K}_{N}$. The set of all vectors $\lambda=\left[\lambda_{1} \ldots \lambda_{N}\right]^{\prime}$ such that $\lambda_{i} \geq 0$, $i \in \mathbb{K}_{N}$ and $\lambda_{1}+\lambda_{2}+\cdots+\lambda_{N}=1$ is designated by $\Lambda_{N}$. The convex combination of a set of matrices $\left(A_{1}, \ldots, A_{N}\right)$ is denoted by $A_{\lambda}=\sum_{i=1}^{N} \lambda_{i} A_{i}$, where $\lambda \in \Lambda_{N}$. In addition, an asterisk ( $*$ ) will be used in matrix expressions to express the transpose of the symmetric element. Moreover, for in-line expressions, the symbol $(*)$ represents the transpose of the left side term. The notation $\operatorname{He}(M)$ refers to $M+M^{\prime}$. The set of all finite $\zeta(t)$ trajectories, such that $\int_{t=0}^{\infty} \zeta(t)^{\prime} \zeta(t) \mathrm{d} t<\infty$ is denoted by $\mathcal{L}_{2}$. For simplicity of notation, $\sigma(t)=\sigma$.

\section{Polytopic uncertain switched systems}

Consider the continuous-time uncertain switched linear system defined by the following state-space realisation:

$$
\begin{aligned}
& \dot{x}(t)=A(\sigma, \alpha) x(t)+B(\sigma, \alpha) u(t)+E(\sigma, \alpha) w(t), \quad x(0)=x_{0}, \\
& z(t)=C_{1}(\sigma, \alpha) x(t)+D(\sigma, \alpha) u(t)+F(\sigma, \alpha) w(t), \\
& y(t)=C_{2}(\alpha) x(t)+H(\alpha) w(t),
\end{aligned}
$$

where $x(t) \in \mathbb{R}^{n_{x}}$ is the state vector, $y(t) \in \mathbb{R}^{n_{y}}$ is the measured output, $z(t) \in \mathbb{R}^{n_{z}}$ is the controlled output, $u(t) \in \mathbb{R}^{n_{u}}$ is the control input, $w(t) \in \mathbb{R}^{n_{w}}$ is an exogenous disturbance input with $w(t) \in \mathcal{L}_{2}[0, \infty)$ and $x_{0}$ is the initial condition. The constant vector $\alpha=\left[\begin{array}{llll}\alpha_{1} & \alpha_{2} & \ldots & \alpha_{r}\end{array}\right]^{\prime}$ represents the polytopic uncertainties of the plant or structural failures (Silva et al., 2013) and (Cardim et al., 2016). Consider that $\sigma(t) \in \mathbb{K}_{N}$ is the switching strategy which selects at each instant of time an available subsystem $i \in \mathbb{K}_{N}$. The matrices $A(\sigma, \alpha), B(\sigma, \alpha), E(\sigma, \alpha), C_{1}(\sigma, \alpha), D(\sigma, \alpha), F(\sigma, \alpha), C_{2}(\alpha)$ and $H(\alpha)$ are constant matrices of appropriate dimensions and can be described by convex combinations of their vertices, as below:

$$
\begin{aligned}
& \left\{\left[A(\sigma, \alpha), \quad B(\sigma, \alpha), \quad E(\sigma, \alpha), \quad C_{1}(\sigma, \alpha), \quad D(\sigma, \alpha),\right.\right. \\
& \left.F(\sigma, \alpha), \quad C_{2}(\alpha), \quad H(\alpha)\right] \\
& \left.=\sum_{j=1}^{r} \alpha_{j}\left[A_{\sigma j}, B_{\sigma j}, E_{\sigma j}, C_{1 \sigma j}, D_{\sigma j}, F_{\sigma j}, C_{2 j}, H_{j}\right]\right\}=\Delta, \\
& \alpha_{j} \geq 0, \quad \sum_{j=1}^{r} \alpha_{j}=1, \quad j \in \mathbb{K}_{r}, \quad \sigma \in \mathbb{K}_{N},
\end{aligned}
$$

where $r$ is the number of vertices of the polytope and $N$ is the number os subsystems.

\section{Robust control of polytopic uncertain switched linear systems based on output-dependent switching}

In practical applications, the state vector may not be completely available. In these cases, it is important to propose switching strategies based on the measured output of the plant. Therefore, this section is devoted to present results concerning the output-dependent switching control.

In this section, the plant equations are given by the switched linear system (1) and (2), but without control input $(u(t)=0$, $t \geq 0)$, without exogenous disturbance $(w(t)=0, t \geq 0)$ and assuming constant output matrices $C_{2 j}=C$, for all $j \in \mathbb{K}_{r}$, as in Mainardi Júnior et al. (2015).

Assume that the state vector $x(t) \in \mathbb{R}^{n_{x}}$ is not completely available, but $y(t) \in \mathbb{R}^{n_{y}}$ is accessible for feedback.

Problem 3.1: Determine a switching strategy $\sigma(\cdot): \mathbb{R}^{p} \rightarrow$ $\{1,2, \ldots, N\}$ such that:

$$
\sigma(t)=f(y(t)), \quad \text { forall } t \geq 0
$$

which makes the origin $(x=0)$ of the polytopic uncertain switched linear system (1) and (2), supposing that $u(t)=0$ and $w(t)=0$ for $t \geq 0$, and assuming constant output matrices $C_{2 j}=$ $C$, for all $j \in \mathbb{K}_{r}$, a globally asymptotically stable equilibrium point.

Mainardi Júnior et al. (2015) presented less conservative conditions for Problem 3.1, described in Theorem 3.1, than conditions available in the literature.

Theorem 3.1 (Mainardi Júnior et al., 2015): If there exist $\lambda \in$ $\Lambda_{N}$, matrices $X_{1_{i k}} \in \mathbb{R}^{n_{x} \times n_{x}}, X_{2_{i k}} \in \mathbb{R}^{n_{x} \times n_{x}}$, symmetric matrices $Q_{0 j k} \in \mathbb{R}^{n_{x} \times n_{x}}, Q_{i} \in \mathbb{R}^{n_{y} \times n_{y}}$ and symmetric positive definite matrices $P_{j k} \in \mathbb{R}^{n_{x} \times n_{x}}$, such that

$$
\begin{gathered}
{\left[\begin{array}{c}
X_{1_{i k}} A_{i j}+A_{i j}^{\prime} X_{1_{i k}}^{\prime}+X_{1_{i j}} A_{i k}+A_{i k}^{\prime} X_{1_{i j}}^{\prime} \\
P_{j k}-X_{1_{i k}}^{\prime}+X_{2_{i k}} A_{i j}+P_{k j}-X_{1_{i j}}^{\prime}+X_{2_{i j}} A_{i k} \\
P_{j k}-X_{1_{i k}}+A_{i j}^{\prime} X_{2_{i k}}^{\prime}+P_{k j}-X_{1_{i j}}+A_{i k}^{\prime} X_{2_{i j}}^{\prime} \\
-X_{2_{i k}}-X_{2_{i k}}^{\prime}-X_{2_{i j}}-X_{2_{i j}}^{\prime}
\end{array}\right]} \\
<\left[\begin{array}{cc}
Q_{0 j k}+Q_{0 k j}+2 C^{\prime} Q_{i} C & 0 \\
0 & 0
\end{array}\right],
\end{gathered}
$$

$$
Q_{0 j k}+C^{\prime} Q_{\lambda} C<0
$$

for all $i \in \mathbb{K}_{N}, j \in \mathbb{K}_{r}$ and $k \in \mathbb{K}_{r}$, then the switching strategy

$$
\sigma(y)=\arg \min _{i \in \mathbb{K}_{N}}\left(y^{\prime} Q_{i} y\right)
$$

makes the origin $x=0$ of the uncertain switched linear system (1) and (2), supposing that $u(t)=0$ and $w(t)=0$ for $t \geq 0$, and assuming constant output matrices $C_{2 j}=C$, for all $j \in \mathbb{K}_{r}$, a globally asymptotically stable equilibrium point.

Proof: See Appendix. 
In order to establish a performance criterion for the switched linear system (1) and (2), the following control problem introduces the guaranteed cost performance (Deaecto et al., 2010), as an extension of Problem 3.1.

Problem 3.2: Determine a switching strategy (3) which makes the origin $x=0$ of the controlled polytopic uncertain switched linear system (1) and (2), supposing that $u(t)=0$ and $w(t)=0$ for $t \geq 0$, and assuming constant output matrices $C_{2 j}=C$, for all $j \in \mathbb{K}_{r}$, a globally asymptotically stable equilibrium point and the guaranteed cost

$$
\begin{aligned}
J & =\int_{0}^{\infty} z(t)^{\prime} z(t) \mathrm{d} t \\
& =\int_{0}^{\infty} x(t)^{\prime} C_{1}(\sigma, \alpha)^{\prime} C_{1}(\sigma, \alpha) x(t) \mathrm{d} t<\mu x_{0}^{\prime} I_{n} x_{0}
\end{aligned}
$$

holds for a given scalar $\mu>0$ and all initial conditions, $x_{0}=$ $x(0) \neq 0$.

A solution of Problem 3.2, that is an extension of the results presented in Mainardi Júnior et al. (2015) for cases where is required a guaranteed cost performance given in (7) and related to the controlled output $z(t)=C_{1}(\sigma, \alpha) x(t)$ defined in (1), is proposed in Theorem 3.2. Furthermore, a specification of the decay rate is also considered in the problem. The decay rate is shown in Boyd, El Ghaoui, Feron, and Balakrishnan (1994) as the largest positive real constant $(\chi)$, such that:

$$
\lim _{t \rightarrow \infty} \mathrm{e}^{\chi t}\|x(t)\|=0
$$

Moreover, if $P(\alpha)=P(\alpha)^{\prime}>0$ is a constant matrix and $V(x(t))=x(t)^{\prime} P(\alpha) x(t)$ is a Lyapunov function for a given system, then the condition $\dot{V}(x(t)) \leq-2 \chi V(x(t))$ assures that the decay rate is greater than or equal to $\chi$.

Theorem 3.2: Consider that there exist $\lambda \in \Lambda_{N}$, a scalar $\mu>$ 0 , matrices $X_{1_{i k}} \in \mathbb{R}^{n_{x} \times n_{x}}, X_{2_{i k}} \in \mathbb{R}^{n_{x} \times n_{x}}$, symmetric matrices $Q_{0 j k} \in \mathbb{R}^{n_{x} \times n_{x}}, Q_{i} \in \mathbb{R}^{n_{y} \times n_{y}}$ and symmetric positive definite matrices $P_{j k} \in \mathbb{R}^{n_{x} \times n_{x}}$, such that

$$
\begin{gathered}
P_{j k}-\mu I_{n}<0 \\
{\left[\begin{array}{c}
X_{1_{i k}} A_{i j}+A_{i j}^{\prime} X_{1_{i k}}^{\prime}+X_{1_{i j}} A_{i k}+A_{i k}^{\prime} X_{1_{i j}}^{\prime}+2 C_{1 i j}^{\prime} C_{1 i k} \\
+2 \chi\left(P_{j k}+P_{k j}\right) P_{j k}-X_{1_{i k}}^{\prime}+X_{2_{i k}} A_{i j}+P_{k j}-X_{1_{i j}}^{\prime}+X_{2_{i j}} A_{i k} \\
P_{j k}-X_{1_{i k}}+A_{i j}^{\prime} X_{i_{i k}}^{\prime}+P_{k j}-X_{1_{i j}}+A_{i k}^{\prime} X_{2_{i j}}^{\prime} \\
-X_{2_{i k}}-X_{2_{i k}}^{\prime}-X_{2_{i j}}-X_{2_{i j}}^{\prime}
\end{array}\right]} \\
<\left[\begin{array}{cc}
Q_{0_{j k}}+Q_{0_{k j}}+2 C^{\prime} Q_{i} C & 0 \\
0 & 0
\end{array}\right] \\
Q_{0_{j k}}+C^{\prime} Q_{\lambda} C<0 .
\end{gathered}
$$

Then, the switching strategy (6) makes the origin $x=0$ of the uncertain switched linear system (1) and (2), supposing that $u(t)=0$ and $w(t)=0$ for $t \geq 0$, and assuming constant output matrices $C_{2 j}=C$, for all $j \in \mathbb{K}_{r}$, a globally asymptotically stable equilibrium point, the decay rate is greater than or equal to $\chi$ and the guaranteed cost (7) holds for all initial conditions, $x_{0}=$ $x(0) \neq 0$.

Proof: Considering that $C_{1 i j}^{\prime} C_{1 i j} \geq 0$ and $2 \chi\left(P_{j k}+P_{k j}\right)>0$, then if (10) and (11) hold, then (4) and (5) also hold. Therefore, from Theorem 3.1, the conditions (10) and (11) assure that the equilibrium point $x=0$ of the controlled system (1), (2), is globally asymptotically stable. The proof of the Theorem 3.1 was performed considering a Lyapunov function $V(x(t))=$ $x(t)^{\prime} P(\alpha) x(t)$, where $P(\alpha)=\sum_{j=1}^{r} \sum_{k=1}^{r} \alpha_{j} \alpha_{k} P_{j k}$ and $P_{j k}=$ $P_{j k}^{\prime}>0$, for all $j, k \in \mathbb{K}_{r}$. Now, following the same steps used in the proof of Theorem 3.1 (Appendix), applied to the conditions (10) and (5), supposing that $C_{1 i j} \neq 0$, from (1), one obtains for $x(t) \neq 0$ :

$$
\begin{aligned}
0> & x(t)^{\prime}\left[\begin{array}{c}
I_{n} \\
A(\sigma, \alpha)
\end{array}\right]^{\prime}\left[\begin{array}{cc}
C_{1}(\sigma, \alpha)^{\prime} C_{1}(\sigma, \alpha)+2 \chi P(\alpha) & P(\alpha) \\
P(\alpha) & 0
\end{array}\right] \\
& \times\left[\begin{array}{c}
I_{n} \\
A(\sigma, \alpha)
\end{array}\right] x(t), \\
0> & \dot{V}(x(t))+2 \chi V(x(t))+z(t)^{\prime} z(t),
\end{aligned}
$$

where $V(x(t))=x(t)^{\prime} P(\alpha) x(t)$ was defined above. Therefore, from (12), $\dot{V}(x(t))<-2 \chi V(x(t))$. Thus, the decay rate is greater than or equal to $\chi$. From (2), note that $V(x(t))>0$ for $x(t) \neq 0$. Observe that, from (1) and (12), $\dot{V}(x(t))<0$ for $x(t) \neq 0$ and thus $x(\infty)=0$. Now, integrating (12) from zero to infinity, considering $x_{0}=x(0) \neq 0$, knowing that $V(x(\infty))=$ 0 , from (9) and remembering that from (2) $\left(\alpha_{1}+\alpha_{2}+\cdots+\right.$ $\left.\alpha_{r}\right)^{2}=1$, it follows that:

$$
J=\int_{0}^{\infty} z(t)^{\prime} z(t) \mathrm{d} t<x_{0}^{\prime} P(\alpha) x_{0}<\mu x_{0}^{\prime} I_{n} x_{0} .
$$

The proof is concluded.

Now, in order to relax the feasibility of the LMIs from Theorem 3.2, based on the results presented in Liu and Zhang (2003), Teixeira et al. (2003), Souza, Teixeira, Cardim, and Assunção (2014), Deaecto, Geromel, and Daafouz (2011) and inspired on the Finsler's lemma (Mozelli \& Palhares, 2011; Qiu, Feng, \& Yang, 2008), less conservative conditions are proposed in Theorem 3.3.

Theorem 3.3: Consider that there exist $\lambda \in \Lambda_{N}$, a scalar $\mu>0$, symmetric matrices $Q_{0_{j k}} \in \mathbb{R}^{n_{x} \times n_{x}}, Q_{i} \in \mathbb{R}^{n_{y} \times n_{y}}$ and matrices $\xi_{i j k}=\xi_{i k j}^{\prime} \in \mathbb{R}^{2 n_{x} \times 2 n_{x}}, \phi_{j k}=\phi_{k j}^{\prime} \in \mathbb{R}^{n_{x} \times n_{x}}, w_{j k}=w_{k j}^{\prime} \in \mathbb{R}^{n_{x} \times n_{x}}$, $P_{j k}=P_{k j}^{\prime} \in \mathbb{R}^{n_{x} \times n_{x}}, X_{1_{i k}} \in \mathbb{R}^{n_{x} \times n_{x}}, X_{2_{i k}} \in \mathbb{R}^{n_{x} \times n_{x}}$, such that

$$
\begin{gathered}
\psi_{i k k}-\gamma_{i k k}<\xi_{i k k}, \\
\psi_{i j k}-\gamma_{i j k}+(*)<\xi_{i j k}+\xi_{i j k}^{\prime}, \quad k \neq j, \\
\theta_{k k}<\phi_{k k}, \\
\theta_{j k}+\theta_{j k}^{\prime}<\phi_{j k}+\phi_{j k}^{\prime}, \quad k \neq j,
\end{gathered}
$$




$$
\begin{gathered}
P_{k k}>w_{k k} \\
P_{j k}+P_{j k}^{\prime}>w_{j k}+w_{j k}^{\prime}, \quad k \neq j, \\
P_{k k}-\mu I_{n}<0, \\
\frac{1}{2} \times\left(P_{j k}+P_{j k}^{\prime}\right)-\mu I_{n}<0, \quad k \neq j, \\
W^{\star}>0, \\
\Phi^{\star}<0, \\
\Xi_{i}^{\star}<0,
\end{gathered}
$$

for all $i \in \mathbb{K}_{N}, j, k \in \mathbb{K}_{r}$, where,

$$
\begin{aligned}
& W^{\star}=\left[\begin{array}{ccc}
w_{11} & \ldots & w_{1 r} \\
\vdots & \ddots & \vdots \\
w_{r 1} & \ldots & w_{r r}
\end{array}\right] \\
& \Phi^{\star}=\left[\begin{array}{ccc}
\phi_{11} & \ldots & \phi_{1 r} \\
\vdots & \ddots & \vdots \\
\phi_{r 1} & \ldots & \phi_{r r}
\end{array}\right] \\
& \Xi_{i}^{\star}=\left[\begin{array}{ccc}
\xi_{i 11} & \ldots & \xi_{i 1 r} \\
\vdots & \ddots & \vdots \\
\xi_{i r 1} & \ldots & \xi_{i r r}
\end{array}\right] \\
& \psi_{i j k}=\left[\begin{array}{cc}
\nu_{i j k} & \vartheta_{i j k} \\
(*) & -X_{2_{i k}}-X_{2_{i k}}^{\prime}-X_{2_{i j}}-X_{2_{i j}}^{\prime}
\end{array}\right], \\
& \gamma_{i j k}=\left[\begin{array}{cc}
Q_{0 j k}+Q_{0_{k j}}+2 C^{\prime} Q_{i} C & 0 \\
0 & 0
\end{array}\right], \\
& \theta_{j k}=Q_{0_{j k}}+C^{\prime} Q_{\lambda} C,
\end{aligned}
$$

$v_{i j k}=X_{1_{i k}} A_{i j}+A_{i j}^{\prime} X_{1_{i k}}^{\prime}+X_{1_{i j}} A_{i k}+A_{i k}^{\prime} X_{1_{i j}}^{\prime}+2 C_{1 i j}^{\prime} C_{1 i k}+2 \chi$

$\left(P_{j k}+P_{k j}\right), \quad \vartheta_{i j k}=P_{j k}-X_{1_{i k}}+A_{i j}^{\prime} X_{2_{i k}}^{\prime}+P_{k j}-X_{1_{i j}}+A_{i k}^{\prime} X_{2_{i j}}^{\prime}$, $Q_{\lambda}=\lambda_{1} Q_{1}+\lambda_{2} Q_{2}+\cdots+\lambda_{N} Q_{N}, \quad \lambda=\left[\begin{array}{ll}\lambda_{1} & \lambda_{2} \ldots \lambda_{N}\end{array}\right]$, $\sum_{i=1}^{N} \lambda_{i}=1$, and $\lambda_{i} \geq 0$ for all $i \in \mathbb{K}_{N}$. Then, the switching strategy (6) makes the origin $x=0$ of the uncertain switched linear system (1) and (2), supposing that $u(t)=0$ and $w(t)=0$ for $t \geq$ 0 , and assuming constant output matrices $C_{2 j}=C$, for all $j \in \mathbb{K}_{r}$, a globally asymptotically stable equilibrium point, the decay rate is greater than or equal to $\chi$ and the guaranteed cost (7) holds for all initial conditions, $x_{0}=x(0) \neq 0$.
Proof: Take into account the following definitions:

$$
\begin{aligned}
X & =\left[\begin{array}{l}
X_{1}(\sigma, \alpha) \\
X_{2}(\sigma, \alpha)
\end{array}\right], \quad T=\left[\begin{array}{c}
I_{n} \\
A(\sigma, \alpha)
\end{array}\right], \\
\sum & =\sum_{j=1}^{r} \alpha_{j} \sum_{k=1}^{r} \alpha_{k}, \quad \Delta(\sigma, \alpha)=\left[\begin{array}{llll}
\alpha_{1} T^{\prime} & \alpha_{2} T^{\prime} & \ldots & \alpha_{r} T^{\prime}
\end{array}\right]^{\prime} .
\end{aligned}
$$

Afterwards, consider that (14)-(24) are feasible. Then, from (14), (15), (26)-(31), it follows that, for $x=x(t) \neq 0$ :

$$
\begin{aligned}
0 & >x^{\prime} \Delta^{\prime}(\sigma, \alpha) \Xi_{i}^{\star} \Delta(\sigma, \alpha) x=x^{\prime} \sum T^{\prime} \xi_{i j k} T x \\
& >x^{\prime} \sum T^{\prime}\left(\psi_{i j k}-\gamma_{i j k}\right) T x .
\end{aligned}
$$

From (28)-(30), note that (32) can be rewritten as:

$$
\begin{aligned}
0 & >x^{\prime} \sum T^{\prime}\left(\left[\begin{array}{cc}
v_{i j k} & \vartheta_{i j k} \\
\vartheta_{i j k}^{\prime} & -X_{2_{i k}}-X_{2_{i k}}^{\prime}-X_{2_{i j}}-X_{2_{i j}}^{\prime}
\end{array}\right]\right. \\
& \left.-\left[\begin{array}{cc}
Q_{0_{j k}}+Q_{0_{k j}}+2 C^{\prime} Q_{i} C & 0 \\
0 & 0
\end{array}\right]\right) T x .
\end{aligned}
$$

Observe that, $\sum\left(X_{1_{i k}} A_{i j}+X_{1_{i j}} A_{i k}\right)=2 \sum X_{1_{i k}} A_{i j}, \quad \sum\left(P_{j k}+\right.$ $\left.P_{k j}\right)=2 \sum P_{j k}, \sum\left(X_{1_{i k}}+X_{1_{i j}}\right)=2 \sum X_{1_{i k}}$ and $\sum\left(Q_{0_{j k}}+Q_{0_{k j}}\right)$ $=2 \sum Q_{0 j k}$. Therefore, verify that (33) can also be described as follows:

$$
\begin{gathered}
0>2 x^{\prime} \sum T^{\prime}\left(\left[\begin{array}{c}
X_{1_{i k}} A_{i j}+(*)+C_{1 i j}^{\prime} C_{1 i k}+2 \chi P_{j k} \\
P_{j k}-X_{1_{i k}}^{\prime}+X_{2_{i k}} A_{i j}
\end{array}\right.\right. \\
\left.\left.\quad-X_{2_{i k}}-X_{2_{i k}}^{\prime}\right]-\left[\begin{array}{cc}
Q_{0_{j k}}+C^{\prime} Q_{i} C & 0 \\
0 & 0
\end{array}\right]\right) T x .
\end{gathered}
$$

Then, define $X_{1}(\sigma, \alpha)=\alpha_{1} X_{1_{\sigma 1}}+\alpha_{2} X_{1_{\sigma 2}}+\cdots+\alpha_{r} X_{1_{\sigma r}}$, $X_{2}(\sigma, \alpha)=\alpha_{1} X_{2_{\sigma 1}}+\alpha_{2} X_{2_{\sigma 2}}+\cdots+\alpha_{r} X_{2_{\sigma r}}, \quad Q_{0}(\alpha)=\alpha_{1} \alpha_{1}$ $Q_{0_{11}}+\alpha_{1} \alpha_{2} Q_{0_{12}}+\alpha_{2} \alpha_{1} Q_{0_{21}}+\cdots+\alpha_{r} \alpha_{r} Q_{0_{r r}}, Q_{i}(\alpha)=\alpha_{1} \alpha_{1}$ $Q_{i_{11}}+\alpha_{1} \alpha_{2} Q_{i_{12}}+\alpha_{2} \alpha_{1} Q_{i_{21}}+\cdots+\alpha_{r} \alpha_{r} Q_{i_{r r}}, \quad C_{1}(\sigma, \alpha)=\alpha_{1}$ $C_{1 \sigma 1}+\alpha_{2} C_{1 \sigma 2}+\ldots+\alpha_{r} C_{1 \sigma r}$ and $P(\alpha)=\alpha_{1} \alpha_{1} P_{11}+\alpha_{1} \alpha_{2}$ $P_{12}+\alpha_{2} \alpha_{1} P_{21}+\cdots+\alpha_{r} \alpha_{r} P_{r r}$. Hence, from (2) and (34), considering (31) and $i=\sigma$ one obtains:

$$
\begin{aligned}
& 0>x^{\prime} T^{\prime}\left(\left[\begin{array}{c}
X_{1}(\sigma, \alpha) A(\sigma, \alpha)+(*)+C_{1}(\sigma, \alpha)^{\prime} C_{1}(\sigma, \alpha) \\
+2 \chi P(\alpha) \\
P(\alpha)-X_{1}^{\prime}(\sigma, \alpha)+X_{2}(\sigma, \alpha) A(\sigma, \alpha)
\end{array}\right.\right.
\end{aligned}
$$

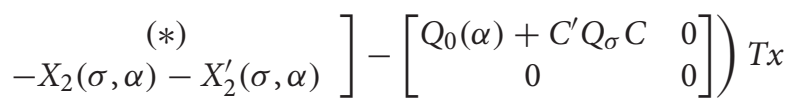

$$
\begin{aligned}
& =x^{\prime} T^{\prime}\left(\left\{\left[\begin{array}{cc}
C_{1}(\sigma, \alpha)^{\prime} C_{1}(\sigma, \alpha)+2 \chi P(\alpha) & P(\alpha) \\
P(\alpha) & 0
\end{array}\right]\right.\right. \\
& \left.+X\left[\begin{array}{c}
A^{\prime}(\sigma, \alpha) \\
-I_{n}
\end{array}\right]^{\prime}+\left[\begin{array}{c}
A^{\prime}(\sigma, \alpha) \\
-I_{n}
\end{array}\right] X^{\prime}\right\} \\
& \left.-\left[\begin{array}{cc}
Q_{0}(\alpha)+C^{\prime} Q_{\sigma} C & 0 \\
0 & 0
\end{array}\right]\right) T x \\
& =x^{\prime} T^{\prime}\left(\left[\begin{array}{cc}
C_{1}(\sigma, \alpha)^{\prime} C_{1}(\sigma, \alpha)+2 \chi P(\alpha) & P(\alpha) \\
P(\alpha) & 0
\end{array}\right]\right. \\
& \left.-\left[\begin{array}{cc}
Q_{0}(\alpha)+C^{\prime} Q_{\sigma} C & 0 \\
0 & 0
\end{array}\right]\right) T x .
\end{aligned}
$$

Now, considering that the minimum of a set of real numbers is less than or equal to an arbitrary convex combination of these 
numbers, observe that, from (1), (2), (6), (30), (31) and defining $V(x(t))=x^{\prime}(t) P(\alpha) x(t),(35)$ becomes:

$$
\begin{aligned}
0 & >\dot{V}(x)+2 \chi V(x)+z^{\prime} z-x^{\prime}\left(Q_{0}(\alpha)\right) x-\min _{i \in \mathbb{K}_{N}}\left(y^{\prime} Q_{i} y\right) \\
& \geq \dot{V}(x)+2 \chi V(x)+z^{\prime} z-x^{\prime} \sum Q_{0_{j k}}+C^{\prime} Q_{\lambda} C x \\
& =\dot{V}(x)+2 \chi V(x)+z^{\prime} z-x^{\prime} \sum \theta_{j k} x
\end{aligned}
$$

Consequently, considering a Lyapunov function candidate $V(x(t))=x(t)^{\prime} P(\alpha) x(t)$, where $P(\alpha)$ was defined above, from (16), (17), (30), note that (36) can be represented as:

$$
\begin{aligned}
0 & >\dot{V}(x)+2 \chi V(x)+z^{\prime} z-\sum_{j=1}^{r} \alpha_{j} \sum_{k=1}^{r} \alpha_{k} x^{\prime} \theta_{j k} x, \\
& >\dot{V}(x)+2 \chi V(x)+z^{\prime} z-\sum_{j=1}^{r} \alpha_{j} \sum_{k=1}^{r} \alpha_{k} x^{\prime} \phi_{j k} x .
\end{aligned}
$$

Now, from (23) and (26), it follows that:

$$
\begin{aligned}
& \dot{V}(x)+2 \chi V(x)+z^{\prime} z<\sum_{j=1}^{r} \alpha_{j} \sum_{k=1}^{r} \alpha_{k} \phi_{j k}, \\
& \dot{V}(x)+2 \chi V(x)+z^{\prime} z<\left[\begin{array}{c}
\alpha_{1} I \\
\alpha_{2} I \\
\ldots \\
\alpha_{r} I
\end{array}\right]^{\prime} \Phi^{\star}\left[\begin{array}{c}
\alpha_{1} I \\
\alpha_{2} I \\
\ldots \\
\alpha_{r} I
\end{array}\right]<0 .
\end{aligned}
$$

Moreover, from (2), (18), (19), (22) and (25), observe that for $x(t) \neq 0$ :

$$
\begin{aligned}
V(x(t)) & =x(t)^{\prime}\left[\begin{array}{c}
\alpha_{1} I \\
\alpha_{2} I \\
\ldots \\
\alpha_{r} I
\end{array}\right]^{\prime}\left[\begin{array}{ccc}
P_{11} & \ldots & P_{1 r} \\
\vdots & \ddots & \vdots \\
P_{r 1} & \ldots & P_{r r}
\end{array}\right]\left[\begin{array}{c}
\alpha_{1} I \\
\alpha_{2} I \\
\ldots \\
\alpha_{r} I
\end{array}\right] x(t), \\
& >x(t)^{\prime}\left[\begin{array}{c}
\alpha_{1} I \\
\alpha_{2} I \\
\ldots \\
\alpha_{r} I
\end{array}\right]^{\prime}\left[\begin{array}{ccc}
w_{11} & \ldots & w_{1 r} \\
\vdots & \ddots & \vdots \\
w_{r 1} & \ldots & w_{r r}
\end{array}\right]\left[\begin{array}{c}
\alpha_{1} I \\
\alpha_{2} I \\
\ldots \\
\alpha_{r} I
\end{array}\right] x(t), \\
& =x(t)^{\prime} \sum_{j=1}^{r} \alpha_{j} \sum_{k=1}^{r} \alpha_{k} W^{\star} x(t)>0 .
\end{aligned}
$$

Furthermore, from (39), note that $V(x(t))>0$, for $x(t) \neq 0$. Now, considering that $P_{j k}=P_{k j}^{\prime}$, from (2), (20) and (21) it follows that $P(\alpha)-\mu I_{n}<0$. Now, from (38), $z(t)^{\prime} z(t) \leq$ $-\dot{V}(x(t))$, and integrating both sides from zero to infinity, considering $x_{0}=x(0) \neq 0$, knowing that, from (38) and (39), the equilibrium point $(x=0)$ of the uncertain switched system (1), (2), and (6) is globally asymptotically stable, then $V(x(\infty))=0$ and one obtains (7). Therefore, the proof is concluded.

Theorem 3.4: If the conditions given in Theorem 3.2 hold, then the conditions given in Theorem 3.3 also hold.

Proof: Considering the definitions (28), (29) and the above conditions, $\psi_{i j k}=\psi_{i j k}^{\prime}, \gamma_{i j k}=\gamma_{i j k}^{\prime}$, then the (10) is equivalent to $\psi_{i j k}-\gamma_{i j k}<0$, for all $i \in \mathbb{K}_{N}$ and $k, j \in \mathbb{K}_{r}$. If (10) holds, then, there exist $\xi_{i k k}=\psi_{i k k}-\gamma_{i k k}+\varepsilon I$, where $\varepsilon>0$, is sufficiently small such that, $\xi_{i k k}<0$, for all $i \in \mathbb{K}_{N}$ and $k \in$ $\mathbb{K}_{r}$, and $\xi_{i j k}=0$ for $k \neq j, i \in \mathbb{K}_{N}, j, k \in \mathbb{K}_{r}$, such that, (14) and (15) hold. Furthermore, in this case, the condition (24) also holds, because it can be rewritten as showed in (27). Note that in Theorem 3.2, $P_{j k}=P_{j k}^{\prime}>0$, but it is not necessary that $P_{j k}=P_{k j}^{\prime}$, for $j \neq k$. However, observe that one can also rewrite $P(\alpha)$ as $P(\alpha)=\sum_{j=1}^{r} \alpha_{j}^{2} P_{j j}+\frac{1}{2} \sum_{j \neq k}^{r} \alpha_{j} \alpha_{k}\left(P_{j k}+\right.$ $\left.P_{k j}\right)=\sum_{j=1}^{r} \alpha_{j}^{2} P_{j j}+\sum_{j \neq k}^{r} \alpha_{j} \alpha_{k} P_{N_{j k}}$, where $P_{N_{j k}}=P_{N_{k j}}^{\prime}=\frac{1}{2} \times$ $\left(P_{j k}+P_{k j}\right)$, because from Theorem 3.2 $P_{j k}=P_{j k}^{\prime}$ for all $j, k \in$ $\mathbb{K}_{r}$. Furthermore, in the LMI (10) given in Theorem 3.2, note that $P_{j k}$ appears added to $P_{k j}$ and then, $P_{j k}+P_{k j}=P_{N_{j k}}+P_{N_{k j}}$. From Theorem 3.2 it also follows that $P_{j k}>0$ for all $j, k \in \mathbb{K}_{r}$. Then, note that, without loss of generality, one can consider in Theorem 3.2 that $P_{j k}=P_{k j}^{\prime}$. Therefore, there exist $w_{k k}=P_{k k}-$ $\varepsilon I$, where $\varepsilon>0$ is sufficiently small such that, $w_{k k}>0$ for all $k \in$ $\mathbb{K}_{r}$, and $w_{j k}=0$ for $j \neq k$, such that the conditions (18) and (19) hold. In this situation, the condition (22) also holds, because it can be rewritten as described (25). Now, from definition (30), the condition (11) is equivalent to $\theta_{j k}<0, j, k \in \mathbb{K}_{r}$. Hence, when (11) holds, observe that (16) and (17) also hold for $\phi_{k k}=$ $\theta_{k k}+\varepsilon I$, where $\varepsilon>0$ is sufficiently small such that, $\phi_{k k}<0$, for all $k \in \mathbb{K}_{r}$, and $\phi_{j k}=0$, for $j \neq k$ and $k, j \in \mathbb{K}_{r}$. In this case, the condition (23) also holds, because it can be rewritten as presented in (26). Finally, when (9) holds for some $\mu>0$, the conditions (20) and (21) also hold. The proof is concluded.

Remark 3.1: Note that the terms $Q_{\lambda}=\sum_{i=1}^{N} \lambda_{i} Q_{i}$ presented in the conditions (5), (11) and (30) of the previous theorems can be seen as BMIs since $Q_{i}$ and $\lambda_{i}$ are variables to be found. However, if the parameters $\lambda_{i}$ are set to be known, those conditions are LMIs that can be solved. Further, an example will exploit the optimisation of the parameters $\lambda_{i}$ in order to achieve suboptimal values for the guaranteed cost.

\subsection{Example I}

In order to compare the potentiality of the proposed theorems, is presented in this section a numerical simulation. The example shows a comparison between the feasible region obtained through the conditions of Theorems 3.2 and 3.3. Furthermore, it is presented a comparative study regarding the guaranteed cost (7).

This example was borrowed from Mainardi Júnior et al. (2015). Consider the uncertain system (1) and (2), supposing that $u(t)=0$ and $w(t)=0$ for $t \geq 0$, and assuming constant output matrices $C_{2 j}=C$, for all $j \in \mathbb{K}_{r}$, with $r=2, N=3$, $i \in$ $\{1,2,3\}, j \in\{1,2\}$ and the following matrices given below:

$$
\begin{array}{rlr}
A_{11} & =\left[\begin{array}{ccc}
2 & 1 & 0 \\
3 & -5 & 0 \\
2 & 0 & -2
\end{array}\right], & A_{12}=\left[\begin{array}{ccc}
h_{1} & 1 & 0 \\
3 & -2 & 0 \\
2 & 0 & -2
\end{array}\right], \\
A_{21} & =\left[\begin{array}{ccc}
-5 & -3 & 1 \\
-3 & -2 & 0 \\
0 & 2 & -2
\end{array}\right], & A_{22}=\left[\begin{array}{ccc}
-5 & -6 & 1 \\
-3 & h_{2} & 0 \\
0 & 2 & -2
\end{array}\right],
\end{array}
$$




$$
\begin{aligned}
A_{31} & =\left[\begin{array}{ccc}
0 & 1 & 0 \\
1 & -2 & 0 \\
3 & 0 & -3
\end{array}\right], \quad A_{32}=\left[\begin{array}{ccc}
0 & 1 & -3 \\
1 & -2 & 0 \\
3 & h_{3} & -3
\end{array}\right], \\
C_{21} & =C_{22}=\left[\begin{array}{lll}
0 & 1 & 0 \\
0 & 0 & 1
\end{array}\right] .
\end{aligned}
$$

A comparative study on feasibility analysis and guaranteed cost for some pairs of $h_{1}$ and $h_{2}$, where $h_{1} \in[-10,0], h_{2} \in$ $[-50,0]$ and $h_{3}=-(h 1+h 2) / 3$ is performed in this example. It was considered increments of 0.5 and 2 regarding the variables $h_{1}$ and $h_{2}$, respectively. Note that the matrices $A_{12}$, $A_{22}$ and $A_{32}$ depend on the parameters $h_{1}, h_{2}$, and $h_{3}$, respectively. Observe that the matrix $A_{12}$ is Hurwitz (i.e. it has all eigenvalues with negative real parts) for all $h_{1} \in[-10,-2]$ and it is not Hurwitz for all $h_{1} \in[-1.5,0]$. The matrix $A_{22}$ is Hurwitz for all $h_{2} \in[-50,-4]$ and it is not Hurwitz for all $h_{2} \in[-2,0]$. Moreover, verify that the matrix $A_{32}$ is Hurwitz for all $h_{3} \in[0,18]$ and it is not Hurwitz for all $h_{2} \in[18.5,20]$. Furthermore, note that the matrices $A_{11}$ and $A_{31}$ are not Hurwitz and the matrix $A_{21}$ is Hurwitz. It was considered $\lambda_{1}=0.4$, $\lambda_{2}=0.3$, and $\lambda_{3}=0.3$. In this example the condition regarding the guaranteed cost (7) is removed, adopting $C_{111}=C_{112}=$ $C_{121}=C_{122}=C_{131}=C_{132}=0$. Thus, note that the conditions from Theorem 3.2 are equivalent to the conditions presented on Theorem 5 (Mainardi Júnior et al., 2015). Figure 1 shows a comparison between the feasible regions obtained through the conditions of Theorems 3.2 and 3.3. Note that, the proposed methodology (Theorem 3.3) presents a greater feasible region than that obtained with the conditions given in (Theorem 5) (Mainardi Júnior et al., 2015). This fact and the result presented in Theorem 3.4 show that the conditions proposed in Theorem 3.3 are less conservative than that presented in Mainardi Júnior et al. (2015).

Furthermore the potentiality of proposed theorems are compared establishing the guaranteed cost as performance criterion, considering $x_{0}=x(0)=[-0,250,5-0,75]^{\prime}, C_{111}=C_{112}=$ $C_{121}=C_{122}=C_{131}=C_{132}=C_{21}=C_{22}$, for $h_{1} \in[-10,-7]$ and $h_{2} \in[-30,-12]$. Observe that, from Figure 2, the less conservative conditions proposed in Theorem 3.3 reduce the guaranteed cost when compared to the conditions of Theorem 3.2. Additionally, note that, when $h_{2} \leq-17$, the conditions of Theorem 3.2 are unfeasible.

\section{Robust switching SOF $\mathscr{H}_{\infty}$ control of continuous time switched linear systems}

This section is devoted to coping with the problem of designing robust SOF $\mathscr{H}_{\infty}$ switching controllers for switched linear uncertain systems. The output feedback controller for the uncertain switched system (1) and (2) is given by

$$
u(t)=\mathcal{K} y(t) .
$$

Consequently, the closed-loop system results in

$$
\begin{aligned}
\dot{x}(t) & =\widetilde{A}(\sigma, \alpha) x(t)+\widetilde{E}(\sigma, \alpha) w(t), \quad x(0)=x_{0} \\
z(t) & =\widetilde{C}_{1}(\sigma, \alpha) x(t)+\widetilde{F}(\sigma, \alpha) w(t), \\
y(t) & =C_{2}(\alpha) x(t)+H(\alpha) w(t),
\end{aligned}
$$

where,

$$
\begin{aligned}
\widetilde{A}(\sigma, \alpha) & =A(\sigma, \alpha)+B(\sigma, \alpha) \mathcal{K} C_{2}(\alpha), \\
\widetilde{E}(\sigma, \alpha) & =E(\sigma, \alpha)+B(\sigma, \alpha) \mathcal{K} H(\alpha), \\
\widetilde{C_{1}}(\sigma, \alpha) & =C_{1}(\sigma, \alpha)+D(\sigma, \alpha) \mathcal{K} C_{2}(\alpha), \\
\widetilde{F}(\sigma, \alpha) & =F(\sigma, \alpha)+D(\sigma, \alpha) \mathcal{K} H(\alpha) .
\end{aligned}
$$

Initially, the approach presented in Chang et al. (2015) is introduced. These results are important to develop the contribution of this section. The following lemma was presented in Chang et al. (2015) and employed to establish the main result of the paper.

Lemma 4.1 (Chang et al., 2015): For matrices $\mathcal{T}, \mathcal{P}, \mathcal{U}$ and $\mathcal{A}$ with appropriate dimension and a scalar $\beta$, the following statements are equivalent:

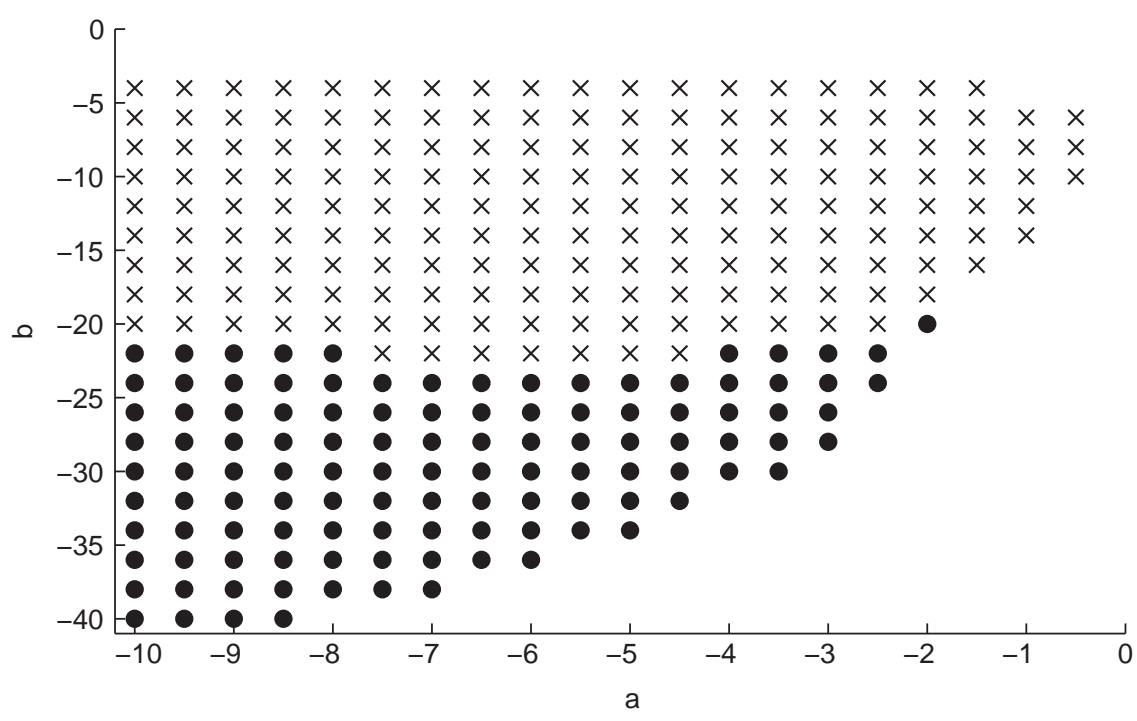

Figure 1. Feasible regions obtained with Theorems 3.2 and 3.3 without the guaranteed cost specification, where the region obtained with Theorem 3.2 is illustrated by $(x)$ and the region obtained for Theorem 3.3 is illustrated by $(x)$ and $(\bullet)$. 


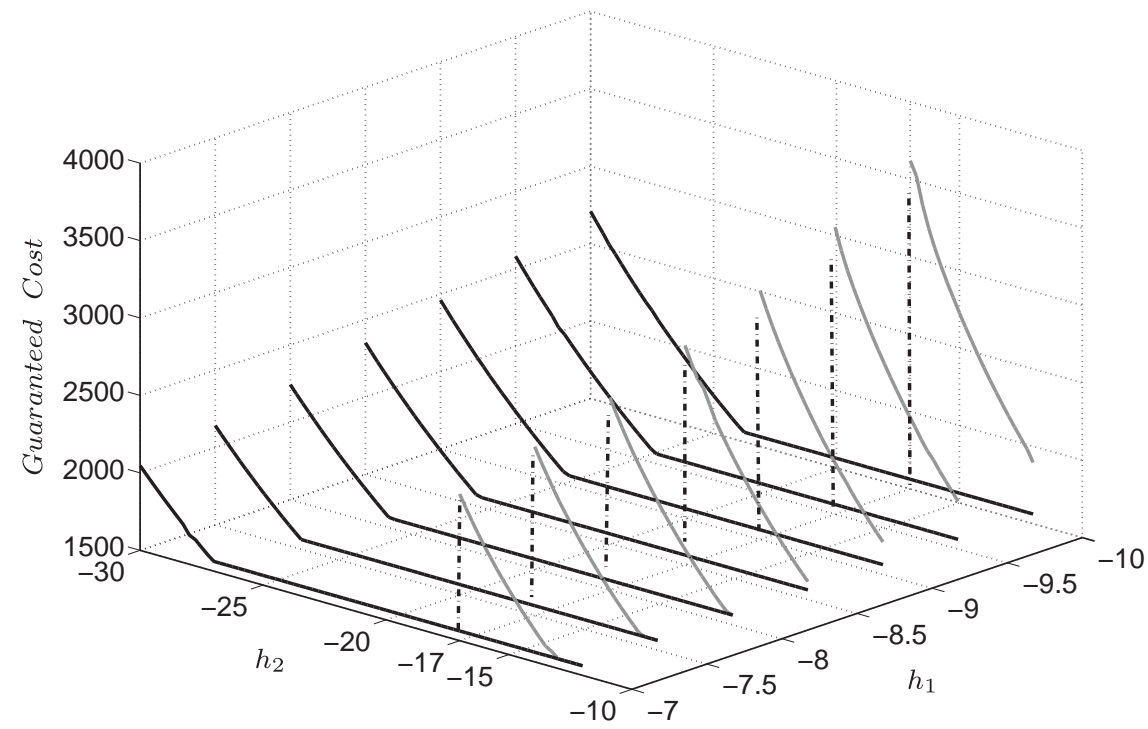

Figure 2. Guaranteed cost obtained from conditions of Theorems 3.2 (gray) and 3.3 (black).

(i): $\left[\begin{array}{c}\mathcal{T} \\ \beta \mathcal{P}^{\prime}+\mathcal{U} \mathcal{A}-\beta \mathcal{U}^{*}-\beta \mathcal{U}^{\prime}\end{array}\right]<0$,

(ii): $\mathcal{T}<0, \mathcal{T}+\mathcal{A}^{\prime} \mathcal{P}^{\prime}+\mathcal{A} \mathcal{P}<0$.

Theorem 4.2 provides LMI-based conditions for designing a SOF $\mathscr{H}_{\infty}$ controller.

Theorem 4.2 (Chang et al., 2015): Given a scalar $\gamma>0$, for known scalar parameters $\beta$ and $\rho$, if there exist matrices $V \in$ $\mathbb{R}^{n_{u} \times n_{y}}, U \in \mathbb{R}^{n_{y} \times n_{y}}$, and $X_{j}>0 \in \mathbb{R}^{n_{x} \times n_{x}}, j=1,2, \ldots, r$ satisfying the following LMIs:

$$
\begin{gathered}
\Omega_{j j}<0, \\
\Omega_{j k}+\Omega_{k j}<0, \quad j<k,
\end{gathered}
$$

for all $j, k \in \mathbb{K}_{r}$, with:

$$
\begin{gathered}
\Omega_{j k}=\left[\begin{array}{cc}
H e\left(A_{j} X_{k}+B_{j} V \mathcal{F}^{k}\right) & * \\
E_{j}^{\prime}+H_{k}^{\prime} V^{\prime} B_{j}^{\prime} & -\gamma^{2} I \\
C_{1 j} X_{k}+\rho \mathcal{F}_{0}^{\prime} V^{\prime} B_{j}^{\prime}+D_{j}^{\prime} V \mathcal{F}^{k} & F_{j}+D_{j} V H_{k} \\
\beta V^{\prime} B_{j}^{\prime}+C_{2 j} X_{k}-U \mathcal{F}^{k} & H_{j}-U H_{k} \\
* & * \\
* & * \\
-I+H e\left(\rho D_{j} V \mathcal{F}_{0}\right) & * \\
\beta V^{\prime} D_{j}^{\prime}-\rho U \mathcal{F}_{0} & -\beta U-\beta U^{\prime}
\end{array}\right] \\
\mathcal{F}^{k}=\left\{\begin{array}{cc}
\left(C_{2} C_{2}^{\prime}\right)^{-1} C_{2}, & C_{2}(\alpha) \text { is fixed, } \quad C_{2}(\alpha)=C_{2} \quad \text { and } \\
C_{2}, \quad C_{2} \text { is of full rank, } & C_{2}(\alpha) \text { is fixed, } \quad C_{2}(\alpha)=C_{2} \text { and } \\
C_{2} \text { is of non }- \text { full rank, } & C_{2}(\alpha) \text { isnon }- \text { fixed, }
\end{array}\right.
\end{gathered}
$$

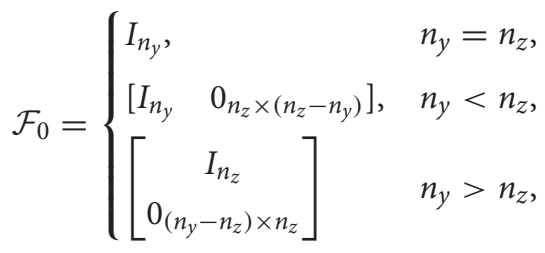

then the system (1) and (2) is asymptotically stable with the $\mathscr{H}_{\infty}$ performance $\gamma$ and the controller gain matrix (41) is given by

$$
\mathcal{K}=K=V U^{-1} .
$$

Proof: See Chang et al. (2015).

Remark 4.1: In Theorem 4.2 if $\rho$ and $\beta$ are set to be known the conditions became LMI which, when feasible, can be easily solved. As mentioned in Chang et al. (2015), these parameters are not necessary but they provide extra free dimensions for the design problem. Therefore it is possible to use numerical optimisation to search suboptimal values for $\beta$ and $\rho$ to reduce the $\mathscr{H}_{\infty}$ bound. In Chang et al. (2015) the function fminsearch of the Matlab optimisation toolbox (Gahinet, Nemirovskii, Laub, \& Chilali, 1994) was used to obtain a locally convergent solution.

In order to extend the conditions of Theorem 4.2 for a class of switched systems and also aiming to design SOF $\mathscr{H}_{\infty}$ switching controllers for linear uncertain systems, the following problem is stated.

Problem 4.1: Find a function $f(\cdot): \mathbb{R}^{n_{y}} \rightarrow[\{1,2, \ldots, N\}$, $\{1,2, \ldots, r\}]$ and gains $K_{i s} \in \mathbb{R}^{m \times p}, i \in \mathbb{K}_{N}, s \in \mathbb{K}_{r}$, such that the switching strategy

$$
\varphi(t)=f(y(t))=\left[\begin{array}{ll}
\sigma(y) & \eta(y)
\end{array}\right], \quad \text { forall } t \geq 0,
$$

and the control input (41), with $\mathcal{K}=K_{\varphi}$, make the origin $x=0$ of the controlled polytopic uncertain switched linear system (1) and 
(2) a globally asymptotically stable equilibrium point such that the controlled system satisfies,

$$
\int_{0}^{\infty} z(t)^{\prime} z(t) \mathrm{d} t \leq \gamma^{2} \int_{0}^{\infty} w(t)^{\prime} w(t) \mathrm{d} t .
$$

Therefore, the $\mathscr{H}_{\infty}$ norm of the aforementioned system is less than $\gamma$ (Dong \& Yang, 2013) for $x(0)=0$ and $w(t) \in \mathcal{L}_{2}[0, \infty]$.

To deal with Problem 4.1, Theorem 4.3 provides conditions for designing static output $\mathscr{H}_{\infty}$ switching controllers for the switched linear uncertain system (1)-(2), based on the results presented in Mainardi Júnior et al. (2015) (Theorem 3.1), Chang et al. (2015) (Theorem 4.2) and Souza et al. (2014).

Theorem 4.3: Consider that for known scalar parameters $\beta$ and $\rho$ there exist $\lambda \in \Lambda$, a scalar $\gamma>0$, matrices $V_{i s} \in \mathbb{R}^{n_{u} \times n_{y}}$, $U_{i s} \in \mathbb{R}^{n_{y} \times n_{y}}, Q_{0 j} \in \mathbb{R}^{n_{x} \times n_{x}}, Q_{1 j} \in \mathbb{R}^{n_{x} \times n_{w}}, Q_{2 j} \in \mathbb{R}^{n_{w} \times n_{w}}$, and symmetric matrices $X_{j}>0 \in \mathbb{R}^{n_{x} \times n_{x}}, \mathcal{Z}_{\text {is }} \in \mathbb{R}^{n_{y} \times n_{y}}$, such that

$$
\begin{gathered}
W_{i j j s}<0, \\
W_{i j k s}+W_{i k j s}<0, \quad j<k \\
\Theta_{\lambda j j j}<0, \\
\Theta_{\lambda j j k}+\Theta_{\lambda j k j}+\Theta_{\lambda k j j}<0, \quad j \neq k \\
\Theta_{\lambda j k q}+\Theta_{\lambda j q k}+\Theta_{\lambda k j q}+\Theta_{\lambda k q j}+\Theta_{\lambda q j k}+\Theta_{\lambda q k j}<0, \\
j<k, k<q .
\end{gathered}
$$

for all $i \in \mathbb{K}_{N}, j, s, k, q \in \mathbb{K}_{r}$, where

$$
\begin{gathered}
W_{i j k s}=\left[\begin{array}{cc}
\operatorname{He}\left(A_{i j} X_{k}+B_{i j} V_{i s} \mathcal{F}^{k}\right)-Q_{0 j}-C_{2 j}^{\prime} \mathcal{Z}_{i s} C_{2 k} \\
E_{i j}^{\prime}+H_{k}^{\prime} V_{i s}^{\prime} B_{i j}^{\prime}-Q_{1 j}^{\prime}-H_{j}^{\prime} \mathcal{Z}_{i s} C_{2 k} \\
C_{1 i j} X_{k}+\rho \mathcal{F}_{0}^{\prime} V_{i s}^{\prime} B_{i j}^{\prime}+D_{i j}^{\prime} V_{i s} \mathcal{F}^{k} \\
\beta V_{i s}^{\prime} B_{i j}^{\prime}+C_{2 j} X_{k}-U_{i s} \mathcal{F}^{k} \\
* & * \\
* & * \\
-\gamma^{2} I-Q_{2 j}-H_{j}^{\prime} \mathcal{Z}_{i s} H_{k} & -I+\operatorname{He}\left(\rho D_{i j} V_{i s} \mathcal{F}_{0}\right) \\
F_{i j}+D_{i j} V_{i s} H_{k} & \beta V_{i s}^{\prime} D_{i j}^{\prime}-\rho U_{i s} \mathcal{F}_{0} \\
H_{j}-U_{i s} H_{k} & \\
* \\
* \\
* \\
-\beta U_{i s}-\beta U_{i s}^{\prime}
\end{array}\right] \\
\Theta_{\lambda j k q}=\left[\begin{array}{cc}
Q_{1 j}+C_{2 j}^{\prime} \mathcal{Z}_{\lambda k} C_{2 q} & Q_{1 j}+C_{2 j}^{\prime} \mathcal{Z}_{\lambda k} H_{q} \\
Q_{1 j}^{\prime}+H_{j}^{\prime} \mathcal{Z}_{\lambda k} C_{2 q} & Q_{2 j}+H_{j}^{\prime} \mathcal{Z}_{\lambda k} H_{q}
\end{array}\right] \\
\mathcal{Z}_{\lambda}(\alpha)=\sum_{i=1}^{N} \sum_{k=1}^{r} \alpha_{k} \lambda_{i} \mathcal{Z}_{i k}
\end{gathered}
$$

with $\mathcal{F}^{k}$ and $\mathcal{F}_{0}$ are given in (47) and (48), respectively.
Thus the switching strategy,

$$
\varphi=\left[\begin{array}{ll}
\sigma & \eta
\end{array}\right]=\arg \min _{\substack{i \in \mathbb{K}_{N} \\
s \in \mathbb{K}_{r}}}\left(y^{\prime} \mathcal{Z}_{i s} y\right)
$$

and the control input (41), with $\mathcal{K}=K_{\phi}=K_{\sigma \eta}$, where the controller gains are given by

$$
K_{i s}=V_{i s} U_{i s}^{-1}, \quad i \in \mathbb{K}_{N}, s \in \mathbb{K}_{r}
$$

make the closed-loop system (1), (2), (41), (59) and (60) asymptotically stable with the $\mathscr{H}_{\infty}$ performance $\gamma$.

Proof: From (2), (52), (53), (57) and considering $i=\sigma, s=\eta$ one obtains:

$$
\begin{aligned}
& \sum_{j=1}^{r} \sum_{k=1}^{r} \alpha_{j} \alpha_{k} W_{\sigma j k \eta}=\sum_{j=1}^{r} \alpha^{2} W_{\sigma j j \eta}+\sum_{j=1}^{r} \sum_{j<k}^{r} \alpha_{j} \alpha_{k}\left(W_{\sigma j k \eta}+W_{\sigma k j \eta}\right) \\
& =\left[\begin{array}{c}
\operatorname{He}\left(A(\sigma, \alpha) X(\alpha)+B(\sigma, \alpha) V_{\sigma \eta} \mathcal{F}(\alpha)\right)-Q_{0}(\alpha)-C_{2}(\alpha)^{\prime} \mathcal{Z}_{\sigma \eta} C_{2}(\alpha) \\
E(\sigma, \alpha)^{\prime}+H(\alpha)^{\prime} V_{\sigma \eta}^{\prime} B(\sigma, \alpha)^{\prime}-Q_{1}(\alpha)^{\prime}-H(\alpha)^{\prime} \mathcal{Z}_{\sigma \eta} C_{2}(\alpha) \\
C_{1}(\sigma, \alpha) X(\alpha)+\rho \mathcal{F}_{0}^{\prime} V_{\sigma \eta}^{\prime} B(\sigma, \alpha)^{\prime}+D(\sigma, \alpha)^{\prime} V_{\sigma \eta} \mathcal{F}(\alpha) \\
\beta V_{\sigma \eta}^{\prime} B(\sigma, \alpha)^{\prime}+C_{2}(\alpha) X(\alpha)-U_{\sigma \eta} \mathcal{F}(\alpha)
\end{array}\right. \\
& \begin{array}{cc}
* & * \\
-\gamma^{2} I-Q_{2}(\alpha)-H(\alpha)^{\prime} \mathcal{Z}_{\sigma \eta} H(\alpha) & *
\end{array} \\
& F(\sigma, \alpha)+D(\sigma, \alpha) V_{\sigma \eta} H(\alpha) \quad-I+\operatorname{He}\left(\rho D(\sigma, \alpha) V_{\sigma \eta} \mathcal{F}_{0}\right) \\
& H(\alpha)-U_{\sigma \eta} H(\alpha) \quad \beta V_{\sigma \eta}^{\prime} D(\sigma, \alpha)^{\prime}-\rho U_{\sigma \eta} \mathcal{F}_{0} \\
& \left.\begin{array}{c}
* \\
* \\
* \\
-\beta U_{\sigma \eta}-\beta U_{\sigma \eta}^{\prime}
\end{array}\right]<0 .
\end{aligned}
$$

Since the inequalities (52) and (53) are feasible, it implies from (57) that $U_{i s}$ are no singular, for all $i \in \mathbb{K}_{N}$ and $r \in \mathbb{K}_{r}$. From Lemma 4.1 with $\mathcal{A}=U_{\sigma \eta}^{-1}\left[C_{2}(\alpha) X(\alpha)-U_{\sigma \eta} \mathcal{F}(\alpha) H(\alpha)-\right.$ $\left.U_{\sigma \eta} H(\alpha)-\rho U_{\sigma \eta} \mathcal{F}_{0}\right], \mathcal{P}^{\prime}=\left[V_{\sigma \eta}^{\prime} B(\sigma, \alpha)^{\prime} 0 V_{\sigma \eta}^{\prime} D(\sigma, \alpha)^{\prime}\right]$ and

$$
\begin{aligned}
& \mathcal{T}=\left[\begin{array}{c}
\operatorname{He}\left(A(\sigma, \alpha) X(\alpha)+B(\sigma, \alpha) V_{\sigma \eta} \mathcal{F}(\alpha)\right)-Q_{0}(\alpha)-C_{2}(\alpha)^{\prime} \mathcal{Z}_{\sigma \eta} C_{2}(\alpha) \\
E(\sigma, \alpha)^{\prime}+H(\alpha)^{\prime} V_{\sigma \eta}^{\prime} B(\sigma, \alpha)^{\prime}-Q_{1}(\alpha)^{\prime}-H(\alpha)^{\prime} \mathcal{Z}_{\sigma \eta} C_{2}(\alpha) \\
C_{1}(\sigma, \alpha) X(\alpha)+\rho \mathcal{F}_{0}^{\prime} V_{\sigma \eta}^{\prime} B(\sigma, \alpha)^{\prime}+D(\sigma, \alpha)^{\prime} V_{\sigma \eta} \mathcal{F}(\alpha)
\end{array}\right. \\
& \left.\begin{array}{cc}
* & * \\
-\gamma^{2} I-Q_{2}(\alpha)-H(\alpha)^{\prime} \mathcal{Z}_{\sigma \eta} H(\alpha) & * \\
F(\sigma, \alpha)+D(\sigma, \alpha) V_{\sigma \eta} H(\alpha) & -I+\operatorname{He}\left(\rho D(\sigma, \alpha) V_{\sigma \eta} \mathcal{F}_{0}\right)
\end{array}\right],
\end{aligned}
$$

the inequality (61) results in

$$
\begin{aligned}
& {\left[\operatorname{He}\left(A(\sigma, \alpha) X(\alpha)+B(\sigma, \alpha) V_{\sigma \eta} \mathcal{F}(\alpha)\right)-Q_{0}(\alpha)-C_{2}(\alpha)^{\prime} \mathcal{Z}_{\sigma \eta} C_{2}(\alpha)\right.} \\
& E(\sigma, \alpha)^{\prime}+H(\alpha)^{\prime} V_{\sigma \eta}^{\prime} B(\sigma, \alpha)^{\prime}-Q_{1}(\alpha)^{\prime}-H(\alpha)^{\prime} \mathcal{Z}_{\sigma \eta} C_{2 \sigma} \\
& C_{1}(\sigma, \alpha) X(\alpha)+\rho \mathcal{F}_{0}^{\prime} V_{\sigma \eta}^{\prime} B(\sigma, \alpha)^{\prime}+D(\sigma, \alpha)^{\prime} V_{\sigma \eta} \mathcal{F}(\alpha) \\
& \left.\begin{array}{cc}
* & * \\
-\gamma^{2} I-Q_{2}(\alpha)-H(\alpha)^{\prime} \mathcal{Z}_{\sigma \eta} H(\alpha) & * \\
F(\sigma, \alpha)+D(\sigma, \alpha) V_{\sigma \eta} H(\alpha) & -I+\operatorname{He}\left(\rho D(\sigma, \alpha) V_{\sigma \eta} \mathcal{F}_{0}\right)
\end{array}\right] \\
& +\operatorname{He}\left(\left[\begin{array}{c}
B(\sigma, \alpha) V_{\sigma \eta} \\
0 \\
D(\sigma, \alpha) V_{\sigma \eta}
\end{array}\right] U_{\sigma \eta}^{-1}\left[\begin{array}{c}
X(\alpha) C_{2}(\alpha)^{\prime}-\mathcal{F}(\alpha)^{\prime} U_{\sigma \eta}^{\prime} \\
H(\alpha)^{\prime}-H(\alpha)^{\prime} U_{\sigma \eta}^{\prime} \\
-\rho \mathcal{F}_{0}^{\prime} U_{\sigma \eta}^{\prime}
\end{array}\right]\right)<0 .
\end{aligned}
$$


Rewriting (63),

$$
\begin{aligned}
& {\left[\begin{array}{ccc}
\operatorname{He}(A(\sigma, \alpha) X(\alpha)) & * & * \\
E(\sigma, \alpha)^{\prime} & -\gamma^{2} I & * \\
C_{1}(\sigma, \alpha) X(\alpha) & F(\sigma, \alpha) & -I
\end{array}\right]} \\
& +\operatorname{He}\left(\left[\begin{array}{c}
B(\sigma, \alpha) V_{\sigma \eta} \\
0 \\
D(\sigma, \alpha) V_{\sigma \eta}
\end{array}\right] U_{\sigma \eta}^{-1}\left[\begin{array}{c}
\mathcal{F}(\alpha)^{\prime} U_{\sigma \eta}^{\prime} \\
H(\alpha)^{\prime} U_{\sigma \eta}^{\prime} \\
\rho \mathcal{F}_{0}^{\prime} U_{\sigma \eta}^{\prime}
\end{array}\right]\right) \\
& +\mathrm{He}\left(\left[\begin{array}{c}
B(\sigma, \alpha) V_{\sigma \eta} \\
0 \\
D(\sigma, \alpha) V_{\sigma \eta}
\end{array}\right] U_{\sigma \eta}^{-1}\left[\begin{array}{c}
X(\alpha) C_{2}(\alpha)^{\prime}-\mathcal{F}(\alpha)^{\prime} U_{\sigma \eta}^{\prime} \\
H(\alpha)^{\prime}-H(\alpha)^{\prime} U_{\sigma \eta}^{\prime} \\
-\rho \mathcal{F}_{0}^{\prime} U_{\sigma \eta}^{\prime}
\end{array}\right]\right) \\
& -\left[\begin{array}{ccc}
Q_{0}(\alpha)+C_{2}(\alpha)^{\prime} \mathcal{Z}_{\sigma \eta} C_{2}(\alpha) & * & * \\
Q_{1}(\alpha)^{\prime}+H(\alpha)^{\prime} \mathcal{Z}_{\sigma \eta} C_{2 \sigma} & Q_{2}(\alpha)+H(\alpha)^{\prime} \mathcal{Z}_{\sigma \eta} H(\alpha) & * \\
0 & 0 & 0
\end{array}\right] \\
& =\left[\begin{array}{ccc}
\operatorname{He}(A(\sigma, \alpha) X(\alpha)) & * & * \\
E(\sigma, \alpha)^{\prime} & -\gamma^{2} I & * \\
C_{1}(\sigma, \alpha) X(\alpha) & F(\sigma, \alpha) & -I
\end{array}\right] \\
& +\mathrm{He}\left(\left[\begin{array}{c}
B(\sigma, \alpha) V_{\sigma \eta} \\
0 \\
D(\sigma, \alpha) V_{\sigma \eta}
\end{array}\right] U_{\sigma \eta}^{-1}\left[\begin{array}{c}
X(\alpha) C_{2}(\alpha)^{\prime} \\
H(\alpha)^{\prime} \\
0
\end{array}\right]\right) \\
& -\left[\begin{array}{ccc}
Q_{0}(\alpha)+C_{2}(\alpha)^{\prime} \mathcal{Z}_{\sigma \eta} C_{2}(\alpha) & * & * \\
Q_{1}(\alpha)^{\prime}+H(\alpha)^{\prime} \mathcal{Z}_{\sigma \eta} C_{2 \sigma} & Q_{2}(\alpha)+H(\alpha)^{\prime} \mathcal{Z}_{\sigma \eta} H(\alpha) & * \\
0 & 0 & 0
\end{array}\right] \\
& =\left[\begin{array}{c}
\operatorname{He}\left(A(\sigma, \alpha) X(\alpha)+B(\sigma, \alpha) K_{\sigma \eta} C_{2}(\alpha) X(\alpha)\right) \\
E(\sigma, \alpha)^{\prime}+H(\alpha)^{\prime} K_{\sigma \eta}^{\prime} B(\sigma, \alpha)^{\prime} \\
\left(C_{1}(\sigma, \alpha)+D(\sigma, \alpha) K_{\sigma \eta} C_{2}(\alpha)\right) X(\alpha)
\end{array}\right. \\
& \left.\begin{array}{cc}
* & * \\
-\gamma^{2} I & * \\
F(\sigma, \alpha)+D(\sigma, \alpha) K_{\sigma \eta} H(\alpha) & -I
\end{array}\right] \\
& -\left[\begin{array}{ccc}
Q_{0}(\alpha)+C_{2}(\alpha)^{\prime} \mathcal{Z}_{\sigma \eta} C_{2}(\alpha) & * & \\
Q_{1}(\alpha)^{\prime}+H(\alpha)^{\prime} \mathcal{Z}_{\sigma \eta} C_{2}(\alpha) & Q_{2}(\alpha)+H(\alpha)^{\prime} \mathcal{Z}_{\sigma \eta} H(\alpha) & * \\
0 & 0 & 0
\end{array}\right]<0 .
\end{aligned}
$$

Using the Schur complement in (64) and considering (43), one obtains

$$
\begin{gathered}
{\left[\begin{array}{cc}
\operatorname{He}(\widetilde{A}(\sigma, \alpha) X(\alpha))+X(\alpha) \widetilde{C}_{1}(\sigma, \alpha)^{\prime} \widetilde{C}_{1}(\sigma, \alpha) X(\alpha) & * \\
\widetilde{E}(\sigma, \alpha)^{\prime}+\widetilde{F}(\sigma, \alpha)^{\prime} \widetilde{C}_{1}(\sigma, \alpha) X(\alpha) & \widetilde{F}(\sigma, \alpha)^{\prime} \widetilde{F}(\sigma, \alpha)-\gamma^{2} I
\end{array}\right]} \\
-\left[\begin{array}{cc}
Q_{0}(\alpha)+C_{2}(\alpha)^{\prime} \mathcal{Z}_{\sigma \eta} C_{2}(\alpha) & * \\
Q_{1}(\alpha)^{\prime}+H(\alpha)^{\prime} \mathcal{Z}_{\sigma \eta} C_{2}(\alpha) & Q_{2}(\alpha)+H(\alpha)^{\prime} \mathcal{Z}_{\sigma \eta} H(\alpha)
\end{array}\right]<0 .
\end{gathered}
$$

Pre and post-multiplying (65) in both sides by $\left[x(t)^{\prime} w(t)^{\prime}\right]$ and its transpose

$$
\begin{aligned}
& {\left[\begin{array}{l}
x(t) \\
w(t)
\end{array}\right]^{\prime}\left(\left[\begin{array}{cc}
\operatorname{He}(\widetilde{A}(\sigma, \alpha) X(\alpha))+X(\alpha) & \\
\widetilde{C}_{1}(\sigma, \alpha)^{\prime} \widetilde{C}_{1}(\sigma, \alpha) X(\alpha) & * \\
\widetilde{E}(\sigma, \alpha)^{\prime}+\widetilde{F}(\sigma, \alpha)^{\prime} \widetilde{C}_{1}(\sigma, \alpha) X(\alpha) & \widetilde{F}(\sigma, \alpha)^{\prime} \widetilde{F}(\sigma, \alpha)-\gamma^{2} I
\end{array}\right]\right.} \\
& \left.-\left[\begin{array}{cc}
Q_{0}(\alpha)+C_{2}(\alpha)^{\prime} \mathcal{Z}_{\sigma \eta} C_{2}(\alpha) & * \\
Q_{1}(\alpha)^{\prime}+H(\alpha)^{\prime} \mathcal{Z}_{\sigma \eta} C_{2}(\alpha) & Q_{2}(\alpha)+H(\alpha)^{\prime} \mathcal{Z}_{\sigma \eta} H(\alpha)
\end{array}\right]\right)\left[\begin{array}{l}
x(t) \\
w(t)
\end{array}\right] \\
& =\left[\begin{array}{c}
x(t) \\
w(t)
\end{array}\right]^{\prime}\left(\left[\begin{array}{cc}
\operatorname{He}(\widetilde{A}(\sigma, \alpha) X(\alpha))+X(\alpha) & * \\
\widetilde{C}_{1}(\sigma, \alpha)^{\prime} \widetilde{C}_{1}(\sigma, \alpha) X(\alpha) & * \\
\widetilde{E}(\sigma, \alpha)^{\prime}+\widetilde{F}(\sigma, \alpha)^{\prime} \widetilde{C}_{1}(\sigma, \alpha) X(\alpha) & \widetilde{F}(\sigma, \alpha)^{\prime} \widetilde{F}(\sigma, \alpha)-\gamma^{2} I
\end{array}\right]\right. \\
& \left.-\left[\begin{array}{cc}
Q_{0}(\alpha) & * \\
Q_{1}(\alpha)^{\prime} & Q_{2}(\alpha)
\end{array}\right]\right)\left[\begin{array}{l}
x(t) \\
w(t)
\end{array}\right]-\left[\begin{array}{c}
x(t) \\
w(t)
\end{array}\right]^{\prime} \\
& \times\left[\begin{array}{cc}
C_{2}(\alpha)^{\prime} \mathcal{Z}_{\sigma \eta} C_{2}(\alpha) & * \\
H(\alpha)^{\prime} \mathcal{Z}_{\sigma \eta} C_{2}(\alpha) & H(\alpha)^{\prime} \mathcal{Z}_{\sigma \eta} H(\alpha)
\end{array}\right]\left[\begin{array}{l}
x(t) \\
w(t)
\end{array}\right] .
\end{aligned}
$$

Observe that,

$$
\begin{aligned}
& {\left[\begin{array}{c}
x(t) \\
w(t)
\end{array}\right]^{\prime}\left[\begin{array}{cc}
C_{2}(\alpha)^{\prime} \mathcal{Z}_{\sigma \eta} C_{2}(\alpha) & * \\
H(\alpha)^{\prime} \mathcal{Z}_{\sigma \eta} C_{2}(\alpha) & H(\alpha)^{\prime} \mathcal{Z}_{\sigma \eta} H(\alpha)
\end{array}\right]\left[\begin{array}{c}
x(t) \\
w(t)
\end{array}\right]} \\
& =\left[\begin{array}{ll}
x(t)^{\prime} & w(t)^{\prime}
\end{array}\right]\left[\begin{array}{l}
C_{2}(\alpha)^{\prime} \\
H(\alpha)^{\prime}
\end{array}\right] \mathcal{Z}_{\sigma \eta}\left[\begin{array}{ll}
C_{2}(\alpha) & H(\alpha)
\end{array}\right]\left[\begin{array}{l}
x(t) \\
w(t)
\end{array}\right] \\
& =\left(x(t)^{\prime} C_{2}(\alpha)^{\prime}+w(t)^{\prime} H(\alpha)^{\prime}\right) \mathcal{Z}_{\sigma \eta}\left(C_{2}(\alpha) x(t)+H(\alpha) w(t)\right) \\
& =y(t)^{\prime} \mathcal{Z}_{\sigma \eta} y(t) \text {. }
\end{aligned}
$$

From (65)-(67), and considering $x(t) \neq 0$, note that

$$
\begin{aligned}
& {\left[\begin{array}{l}
x(t) \\
w(t)
\end{array}\right]^{\prime}\left(\left[\begin{array}{cc}
\operatorname{He} & (\widetilde{A}(\sigma, \alpha) X(\alpha))+X(\alpha) \\
\widetilde{C}_{1}(\sigma, \alpha)^{\prime} \widetilde{C}_{1}(\sigma, \alpha) X(\alpha) & * \\
\widetilde{E}(\sigma, \alpha)^{\prime}+\widetilde{F}(\sigma, \alpha)^{\prime} \widetilde{C}_{1}(\sigma, \alpha) X(\alpha) & \widetilde{F}(\sigma, \alpha)^{\prime} \widetilde{F}(\sigma, \alpha)-\gamma^{2} I
\end{array}\right]\right.} \\
& \left.-\left[\begin{array}{cc}
Q_{0}(\alpha) & * \\
Q_{1}(\alpha)^{\prime} & Q_{2}(\alpha)
\end{array}\right]\right)\left[\begin{array}{l}
x(t) \\
w(t)
\end{array}\right]-y(t)^{\prime} \mathcal{Z}_{\sigma \eta} y(t)<0
\end{aligned}
$$

Now, from (54)-(56) one has

$$
\begin{aligned}
\sum_{j=1}^{r} \sum_{k=1}^{r} \sum_{q=1}^{r} \alpha_{j} \alpha_{k} \alpha_{q} \Theta_{\lambda j k q} & \\
= & \sum_{j=1}^{r} \alpha_{j}^{3} \Theta_{\lambda j j j}+\sum_{j=1}^{r} \sum_{j \neq k}^{r} \alpha_{j}^{2} \alpha_{k}\left(\Theta_{\lambda j j k}+\Theta_{\lambda j k j}+\Theta_{\lambda j j k}\right) \\
& +\sum_{j=1}^{r} \sum_{j<k}^{r} \sum_{k<q}^{r} \alpha_{j} \alpha_{k} \alpha_{q}\left(\Theta_{\lambda j k q}+\Theta_{\lambda j q k}+\Theta_{\lambda k j q}\right. \\
& \left.+\Theta_{\lambda k q j}+\Theta_{\lambda q j k}+\Theta_{\lambda q k j}\right) \\
= & {\left[\begin{array}{ll}
Q_{0}(\alpha) & Q_{1}(\alpha) \\
Q_{1}(\alpha)^{\prime} & Q_{2}(\alpha)
\end{array}\right] } \\
& +\left[\begin{array}{ll}
C_{2}(\alpha)^{\prime} \mathcal{Z}_{\lambda}(\alpha) H(\alpha) & C_{2}(\alpha)^{\prime} \mathcal{Z}_{\lambda}(\alpha) H(\alpha) \\
H(\alpha)^{\prime} \mathcal{Z}_{\lambda}(\alpha) C_{2}(\alpha) & H(\alpha)^{\prime} \mathcal{Z}_{\lambda}(\alpha) H(\alpha)
\end{array}\right]<0 .
\end{aligned}
$$

Pre and post-multiplying (70) in both sides by $\left[x(t)^{\prime} w(t)^{\prime}\right]$ and its transpose result in

$$
\begin{gathered}
{\left[\begin{array}{l}
x(t) \\
w(t)
\end{array}\right]^{\prime}\left(\left[\begin{array}{ll}
Q_{0}(\alpha) & Q_{1}(\alpha) \\
Q_{1}(\alpha)^{\prime} & Q_{2}(\alpha)
\end{array}\right]+\left[\begin{array}{ll}
C_{2}(\alpha)^{\prime} \mathcal{Z}_{\lambda}(\alpha) H(\alpha) & C_{2}(\alpha)^{\prime} \mathcal{Z}_{\lambda}(\alpha) H(\alpha) \\
H(\alpha)^{\prime} \mathcal{Z}_{\lambda}(\alpha) C_{2}(\alpha) & H(\alpha)^{\prime} \mathcal{Z}_{\lambda}(\alpha) H(\alpha)
\end{array}\right]\right)} \\
{\left[\begin{array}{l}
x(t) \\
w(t)
\end{array}\right]=\left[\begin{array}{l}
x(t) \\
w(t)
\end{array}\right]^{\prime}\left[\begin{array}{ll}
Q_{0}(\alpha) & Q_{1}(\alpha) \\
Q_{1}(\alpha)^{\prime} & Q_{2}(\alpha)
\end{array}\right]\left[\begin{array}{l}
x(t) \\
w(t)
\end{array}\right]+y(t)^{\prime} \mathcal{Z}_{\lambda}(\alpha) y(t)<0 .}
\end{gathered}
$$

Now, knowing that the minimum of a set of real numbers is less than or equal to an arbitrary convex combination of these numbers it is possible to rewrite (68), considering the definition of $\mathcal{Z}_{\lambda}(\alpha)$ in (58):

$$
\begin{aligned}
& 0>-\min _{\substack{i \in \mathbb{K}_{N} \\
s \in \mathbb{K}_{r}}}\left(y^{\prime} \mathcal{Z}_{i s} y\right)+\left[\begin{array}{c}
x(t) \\
w(t)
\end{array}\right]^{\prime}\left(-\left[\begin{array}{cc}
Q_{0}(\alpha) & * \\
Q_{1}(\alpha)^{\prime} & Q_{2}(\alpha)
\end{array}\right]\right. \\
& \left.+\left[\begin{array}{cc}
\operatorname{He}(\widetilde{A}(\sigma, \alpha) X(\alpha)) & \\
+X(\alpha) \widetilde{C}_{1}(\sigma, \alpha)^{\prime} \widetilde{C}_{1}(\sigma, \alpha) X(\alpha) & * \\
\widetilde{E}(\sigma, \alpha)^{\prime}+\widetilde{F}(\sigma, \alpha)^{\prime} \widetilde{C}_{1}(\sigma, \alpha) X(\alpha) & \widetilde{F}(\sigma, \alpha)^{\prime} \widetilde{F}(\sigma, \alpha)-\gamma^{2} I
\end{array}\right]\right)\left[\begin{array}{l}
x(t) \\
w(t)
\end{array}\right] \\
& \geq-y(t)^{\prime} \mathcal{Z}_{\lambda}(\alpha) y(t)-\left[\begin{array}{l}
x(t) \\
w(t)
\end{array}\right]^{\prime}\left[\begin{array}{cc}
Q_{0}(\alpha) & * \\
Q_{1}(\alpha)^{\prime} & Q_{2}(\alpha)
\end{array}\right]\left[\begin{array}{l}
x(t) \\
w(t)
\end{array}\right] \\
& +\left[\begin{array}{c}
x(t) \\
w(t)
\end{array}\right]^{\prime}\left[\begin{array}{c}
\operatorname{He}(\widetilde{A}(\sigma, \alpha) X(\alpha))+X(\alpha) \widetilde{C}_{1}(\sigma, \alpha)^{\prime} \widetilde{C}_{1}(\sigma, \alpha) X(\alpha) \\
\widetilde{E}(\sigma, \alpha)^{\prime}+\widetilde{F}(\sigma, \alpha)^{\prime} \widetilde{C}_{1}(\sigma, \alpha) X(\alpha)
\end{array}\right. \\
& \left.\widetilde{F}(\sigma, \alpha)^{*} \widetilde{F}(\sigma, \alpha)-\gamma^{2} I\right]\left[\begin{array}{l}
x(t) \\
w(t)
\end{array}\right] .
\end{aligned}
$$


Therefore, from (72),

$$
\begin{aligned}
& {\left[\begin{array}{c}
x(t) \\
w(t)
\end{array}\right]^{\prime}\left[\begin{array}{c}
\operatorname{He}(\widetilde{A}(\sigma, \alpha) X(\alpha))+X(\alpha) \widetilde{C}_{1}(\sigma, \alpha)^{\prime} \widetilde{C}_{1}(\sigma, \alpha) X(\alpha) \\
\widetilde{E}(\sigma, \alpha)^{\prime}+\widetilde{F}(\sigma, \alpha)^{\prime} \widetilde{C}_{1}(\sigma, \alpha) X(\alpha)
\end{array}\right.} \\
& \left.\widetilde{F}(\sigma, \alpha)^{\prime} \widetilde{F}(\sigma, \alpha)-\gamma^{2} I\right]\left[\begin{array}{l}
x(t) \\
w(t)
\end{array}\right] \\
& \leq y(t)^{\prime} \mathcal{Z}_{\lambda}(\alpha) y(t)+\left[\begin{array}{c}
x(t) \\
w(t)
\end{array}\right]^{\prime}\left[\begin{array}{cc}
Q_{0}(\alpha) & * \\
Q_{1}(\alpha)^{\prime} & Q_{2}(\alpha)
\end{array}\right]\left[\begin{array}{c}
x(t) \\
w(t)
\end{array}\right] .
\end{aligned}
$$

Considering (71) and (73)

$$
\begin{aligned}
& {\left[\begin{array}{c}
\operatorname{He}(\widetilde{A}(\sigma, \alpha) X(\alpha))+X(\alpha) \widetilde{C}_{1}(\sigma, \alpha)^{\prime} \widetilde{C}_{1}(\sigma, \alpha) X(\alpha) \\
\widetilde{E}(\sigma, \alpha)^{\prime}+\widetilde{F}(\sigma, \alpha)^{\prime} \widetilde{C}_{1}(\sigma, \alpha) X(\alpha)
\end{array}\right.} \\
& \left.\widetilde{F}(\sigma, \alpha)^{*} \widetilde{F}(\sigma, \alpha)-\gamma^{2} I\right]<0 .
\end{aligned}
$$

Considering a Lyapunov function candidate $V(x(t))=$ $x(t) P(\alpha) x(t)$, defining $X(\alpha)=P^{-1}(\alpha)$ and pre- and postmultiplying both sides of (74) with $\operatorname{diag}\{P(\alpha), I\}$ and its transpose and then pre- and post-multiplying the result in both sides with $\left[x(t)^{\prime} w(t)^{\prime}\right]$, one has

$$
\begin{gathered}
{\left[\begin{array}{l}
x(t)^{\prime} \\
w(t)^{\prime}
\end{array}\right]\left[\begin{array}{cc}
\operatorname{He}\left(\widetilde{A}(\sigma, \alpha)^{\prime} P(\alpha)\right)+\widetilde{C}_{1}(\sigma, \alpha)^{\prime} \widetilde{C}_{1}(\sigma, \alpha) & * \\
\widetilde{E}(\sigma, \alpha)^{\prime} P(\alpha)+\widetilde{F}(\sigma, \alpha)^{\prime} \widetilde{C}_{1}(\sigma, \alpha) & \widetilde{F}(\sigma, \alpha)^{\prime} \widetilde{F}(\sigma, \alpha)-\gamma^{2}
\end{array}\right]} \\
{\left[\begin{array}{l}
x(t) \\
w(t)
\end{array}\right]=\dot{V}(t)+z(t)^{\prime} z(t)-\gamma^{2} w(t)^{\prime} w(t)<0 .}
\end{gathered}
$$

Integrating (75) from zero to infinity

$$
\begin{aligned}
\int_{0}^{\infty} & \dot{V}(t) \mathrm{d} t+\int_{0}^{\infty} z(t)^{\prime} z(t) \mathrm{d} t-\gamma^{2} \int_{0}^{\infty} w(t)^{\prime} w(t) \mathrm{d} t \\
= & V(\infty)-V(x(0))+\int_{0}^{\infty} z(t)^{\prime} z(t) \mathrm{d} t \\
& -\gamma^{2} \int_{0}^{\infty} w(t)^{\prime} w(t) \mathrm{d} t<0
\end{aligned}
$$

Considering $x(0)=0$, then $V(x(0))=0$ and $V(\infty) \geq 0$. Therefore, from (76) one has

$$
\int_{0}^{\infty} z(t)^{\prime} z(t) \mathrm{d} t \leq \gamma^{2} \int_{0}^{\infty} w(t)^{\prime} w(t) \mathrm{d} t .
$$

Thus, (51) holds and the $\mathscr{H}_{\infty}$ performance is fulfilled. Note that from (75) if $w(t)=0$ one obtains $\dot{V}(t)<0$ for all $x(t) \neq 0$. Hence, the proof is concluded.

Remark 4.2: As mentioned in Remark 4.1, in Theorem 4.2 to obtain LMI conditions the parameters $\beta$ and $\rho$ are set to be known. In Theorem 4.3 , besides $\beta$ and $\rho$, the parameters $\lambda_{i}, i \in$ $\mathbb{K}_{N}$ need to be known to have LMI conditions. It is important to highlight that the parameters $\lambda_{i}$, unlike $\beta$ and $\rho$, are necessary for the conditions. Obviously, as mentioned in Remark 3.1, the same discussion concerning $\lambda_{i}$ can be drawn for Theorems $3.1-3.3$ since the convex combination of the parameters $\lambda_{i}$ is needed. With the purpose of optimising the $\mathscr{H}_{\infty}$ norm finding the values of $\beta, \rho$ and $\lambda_{i}, i \in \mathbb{K}_{N}$, the DE-LMI algorithm will be introduced later.
Theorem 4.4: If the conditions given in Theorem 4.2 hold, then the conditions given in Theorem 4.3 also hold.

Proof: For Theorem 4.3 assume the particular case where $N=1$ and the controller gains are fixed $(\mathcal{K}=K)$ which yield fixed values for $\mathcal{Z}_{i s}=\mathcal{Z}, U_{i s}=U, V_{i s}=V, \Theta_{\lambda j k q}=\Theta_{j}$ and $W_{i j k s}=$ $W_{j k}$. Therefore, assuming $\mathcal{Z}=0$, it is possible to rewrite (57) as

$$
\begin{gathered}
W_{j k}= \\
+\underbrace{\left[\begin{array}{cccc}
-Q_{0 j} & * & * & * \\
-Q_{1 j}^{\prime} & -Q_{2 j} & * & * \\
0 & 0 & 0 & * \\
0 & 0 & 0 & 0
\end{array}\right]}_{\begin{array}{c}
\operatorname{He}\left(A_{j} X_{k}+B_{j} V \mathcal{F}^{k}\right) \\
E_{j}^{\prime}+H_{k}^{\prime} V^{\prime} B_{j}^{\prime} \\
C_{1 j} X_{k}+\rho \mathcal{F}_{0}^{\prime} V^{\prime} B_{j}^{\prime}+D_{j}^{\prime} V \mathcal{F}^{k} \\
\beta V^{\prime} B_{j}^{\prime}+C_{2 j} X_{k}-U \mathcal{F}^{k}
\end{array}},
\end{gathered}
$$$$
\begin{array}{cc}
* & * \\
-\gamma^{2} I & *
\end{array}
$$$$
\begin{aligned}
& * \\
& * \\
&\left.D_{j} V \mathcal{F}_{0}\right) \quad *
\end{aligned}
$$$$
\left.\begin{array}{ccc}
+D_{j} V H_{k} & -I+\operatorname{He}\left(\rho D_{j} V \mathcal{F}_{0}\right) & * \\
H_{j}-U H_{k} & \beta V^{\prime} D_{j}^{\prime}-\rho U \mathcal{F}_{0} & -\beta U-\beta U^{\prime}
\end{array}\right]
$$$$
\omega_{j k}
$$

and (58) as

$$
\Theta_{j}=\left[\begin{array}{cc}
Q_{0 j} & * \\
Q_{1 j}^{\prime} & Q_{2 j}
\end{array}\right] .
$$

Note that for this particular case the term $\omega_{j k}$ defined in (78) is equal to $\Omega_{j k}$ given in (46) of Theorem 4.2. Considering that the conditions of Theorem 4.2 hold, then from (44) and (45) one has that $\omega_{j j}=\Omega_{j j}<0$ and $\omega_{j k}+\omega_{k j}=\Omega_{j k}+\Omega_{j k}<0$. Thus, there exists sufficient small parameters $\varepsilon>0$ and $\tau>0$ such that $\omega_{j j}+\varepsilon I<0$ and $\omega_{j k}+\omega_{k j}+\tau I<0$. Therefore, from (78), for the particular case where $Q_{0 j}=-\varepsilon I, Q_{2 j}=-\varepsilon I$ and $Q_{1 j}=$ 0 , considering (57), then (52) and (53) hold. Hence (54), (55) and (56) also hold, since $Q_{0 j}=-\varepsilon I, Q_{2 j}=-\varepsilon I$ and $Q_{1 j}=0$, because $\Theta_{\lambda j k q}=\Theta_{j}$ and from (79), $\Theta_{j}=-\varepsilon I<0$. The proof is concluded.

Corollary 4.5: Theorem 4.3 also suits for designing switched output feedback $\mathscr{H}_{\infty}$ controllers for continuous-time uncertain linear systems. These systems can be considered a particular case of the switched systems with $N=1$.

\section{Hybrid DE-LMI-based algorithm}

Note that as mentioned in Remarks 3.1 and 4.2, terms $Q_{\lambda}$ lead to BMIs conditions in Theorems 3.1- 3.3 and 4.3. As well as stated in Remark 4.1, the parameters $\rho$ and $\beta$ in the Theorems 4.2 and 4.3 can be found through numerical optimisation in order to reduce the $\mathscr{H}_{\infty}$ norm. Currently, to the best of the author's knowledge, there are not available solvers (deterministic methods) in literature able to find the optimum solution for non-convex problems (Sadabadi \& Peaucelle, 2016). Thus, finding the output gains in order to stabilise an uncertain switched linear system is a NP-hard problem (Koumboulis \& Tzamtzi, 2007; Lin \& Antsaklis, 2009). Therefore, it is proposed the use of an hybrid metaheuristic technique, DE-LMI (Differential Evolution - Linear Matrix Inequality) (Storn \& Price, 1997) for finding quasi-optimum values for the parameters $\rho, \beta$ and $\lambda_{i}$.

\subsection{Differential evolution}

Global optimisation is considered effective in different fields of engineering, statistics, and finances models. Consequently, 


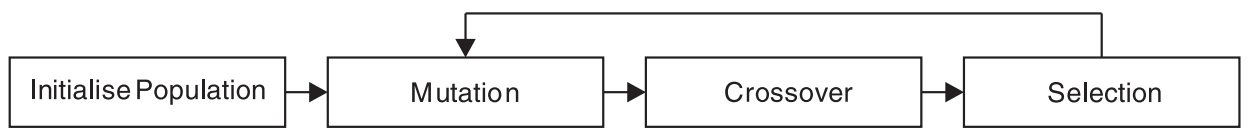

Figure 3. Main stages of DE algorithm (Das \& Suganthan, 2011).

there are different techniques proposed in the literature to solve these problems. DE is a stochastic method based on population optimisation algorithm introduced by Storn and Price (1997). This method belongs to the class of Evolutionary Algorithms (EA), which also includes, Genetic Algorithms (GA), Evolutionary Strategies (ES) and Evolutionary Programming (EP) (Price, Storn, \& Lampinen, 2006). DE figured as one of the best among the competing algorithms presented at Second International Contest on Evolutionary Optimisation and over the the years has attracted the attention of researchers from diverse fields of knowledge. Hence, a plenty of variants of the basic DE algorithm has emerged (Das \& Suganthan, 2011). DE is a real parameter algorithm and can be summarised in four stages, such as GA: initialisation of the population/parameters, mutation, crossover, and selection. Figure 3 depicts these stages.

The notation for representing a population consisting of $N P$ vectors that will be updated throughout the $G$ generations was adopted as following (80):

$$
\left\{\vec{x}_{i, G} \mid i=0,1,2 \ldots N P-2, N P-1\right\} .
$$

To initialise the population $(G=0)$, in order to cover the suitable range with uniformly distributed random individuals, one has to consider the knowledge about the problem. For instance, if the parameters of the convex combination compose the population, knowing that $\lambda_{i} \leq 1, \lambda_{i} \geq 0$ and $\sum_{i=1}^{N} \lambda_{i}=1$, the initial population should cover the range between 0 and 1 , considering the sum of the individuals equal to 1 , with uniformly distributed random values.

The mutation stage involves adding the weighted difference between two random individuals $\left(\vec{x}_{\beta, G}\right.$ and $\left.\vec{x}_{\gamma, G}\right)$ of the population to a third one $\left(\vec{x}_{\kappa, G}\right)$, as shown in (81).

$$
\vec{v}=\vec{x}_{\kappa, G}+F\left(\vec{x}_{\beta, G}-\vec{x}_{\gamma, G}\right),
$$

where the real and constant $F>0$ factor controls the amplification of the differential variation and for each target vector $\vec{x}_{i, G}$ a mutant vector is generated (Storn \& Price, 1997). The vector $\vec{x}_{\kappa, G}$ may be replaced by the best individual in the population at generation $G\left(\vec{x}_{b e s t, G}\right)$. In order to do that, when initialising the population the fitness function must be evaluated.

Aiming to increase the diversity, the crossover between the mutant vector and the target vector is performed to yield the trial vector $(\vec{u})$. The last stage (selection) involves whether or not the trial vector should replace the target vector. The trial vector replaces the target vector if it yields a smaller fitness function. Otherwise, the target vector is held.

\subsection{DE-LMI-based algorithm employed to the proposed problem}

As aforementioned the proposed DE-LMI-based algorithm applies the SeDuMi algorithm (Sturm, 1999) or Matlab LMI toolbox (Gahinet et al., 1994) for solving the LMI problems in order to provide the parameters to evaluate each individual of the population considered in the DE. Considering the DE traditional algorithm described in Section 5.1 and in order to illustrate the integration of DE and LMI solvers, Figure 4 depicts the algorithm routine.

Our main goal is to find the convex combination parameters $\left(\lambda_{i}\right), \beta$ and $\rho$ when required. It is possible to consider that the characteristics of the individuals are the convex combination parameters and those aforementioned parameters. Hence, aiming to minimise the guaranteed cost for Theorems 3.2 and 3.3 and the $\mathscr{H}_{\infty}$ cost for Theorem 4.3, is possible to apply the DE-LMI algorithm to deal with the problem, achieving quasi-optimum values for $\lambda_{i}, \beta$ and $\rho$.

\section{Examples}

In this section, three examples are used to compare the potentiality of the theorems proposed in this paper and complement the results presented in Section 3.1. Example II illustrates the case where all matrices of the subsystems are not Hurwitz. Comparisons regarding the guaranteed cost were performed considering a given set of $\lambda_{1}, \lambda_{2}$ and $\lambda_{3}$ and the suboptimal $\lambda_{1}, \lambda_{2}$ and $\lambda_{3}$ obtained using the proposed hybrid algorithm DE-LMI. Example II, consider that $u(t)=0$ and $w(t)=0$, and thus Theorems 3.2 and 3.3 are compared. With the aim to compare the proposed technique with the results presented in Chang et al. (2015) for continuous-time systems, the condition of Corollary 4.5 is used to cover the Example III. Finally, in Example IV the conditions of Theorem 4.3 are used in order to find a feasible solution for a practical application of the method in the design of a switched robust controller for a semi-active suspension (Cardim et al., 2016) and (Geromel et al., 2008) aiming to minimise the $\mathscr{H}_{\infty}$ cost (51). Concerning LMI solver, it was used the SeDuMi or Matlab LMI toolbox interfaced by YALMIP in MATLAB software.

\subsection{Example II - numerical simulation}

Consider the uncertain switched linear systems represented by (1) and (2), with $r=2, N=3, i \in\{1,2,3\}, j \in\{1,2\}$ and the following matrices given below:

$$
\begin{array}{ll}
A_{1}(a)=\left[\begin{array}{ccc}
a & 1 & 0 \\
1 & -3 & 0 \\
1 & 0 & -1
\end{array}\right], & A_{2}(b)=\left[\begin{array}{ccc}
-1 & -2 & 0 \\
-2 & b & 0 \\
0 & 1 & -1
\end{array}\right], \\
A_{3}(c)=\left[\begin{array}{ccc}
-2 & 0 & 1 \\
0 & -1 & 2 \\
0 & 1 & c
\end{array}\right], & C_{21}=C_{22}=C_{1 i j}=\left[\begin{array}{lll}
1 & 0 & 0 \\
0 & 0 & 1
\end{array}\right] .
\end{array}
$$

Since there neither control input $(u(t)=0)$ nor exogenous disturbance $(w(t)=0)$ the matrices $B_{i j}, E_{i j}, D_{i j}, F_{i j}$ and $H_{j}$ are not 


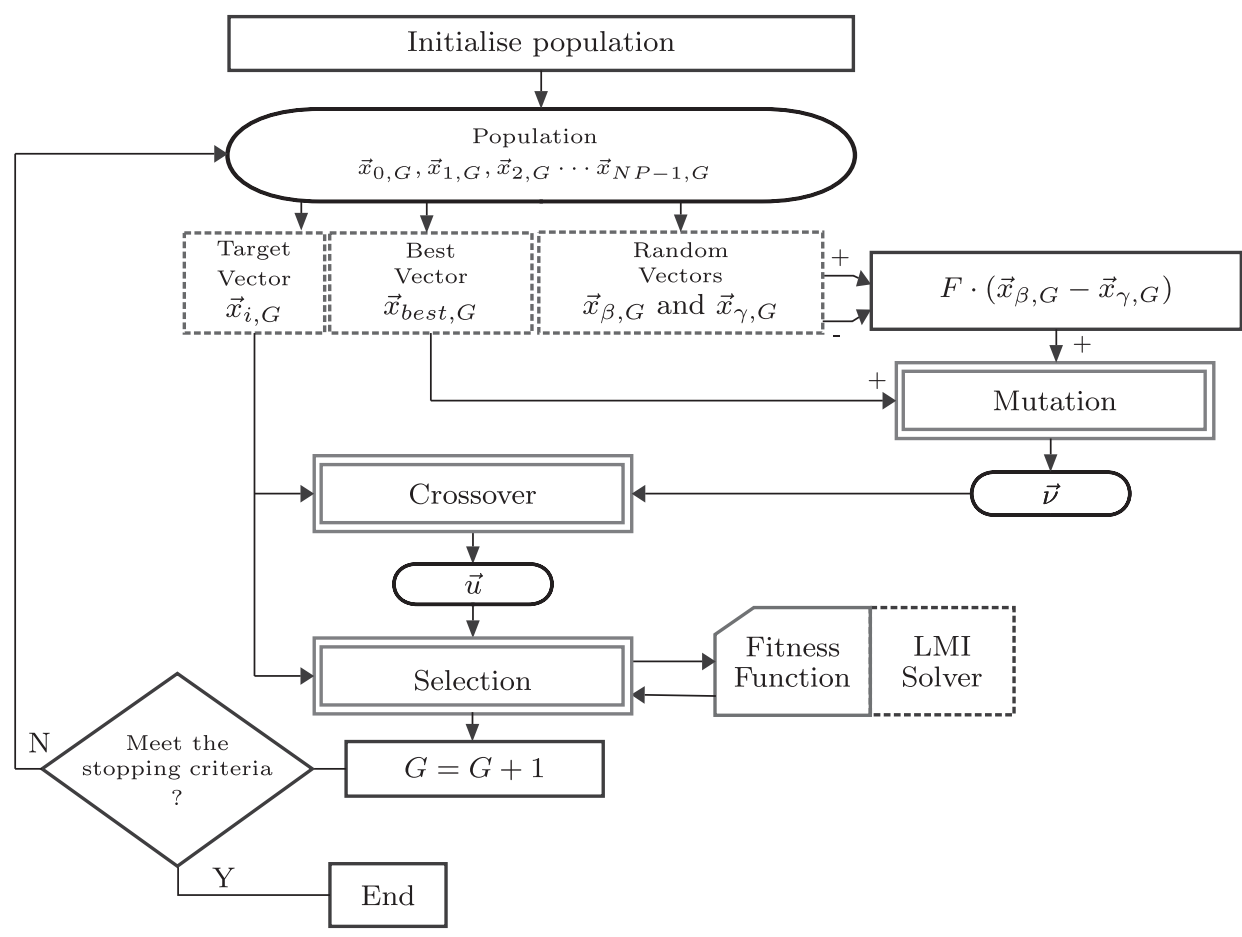

Figure 4. DE-LMI routine.

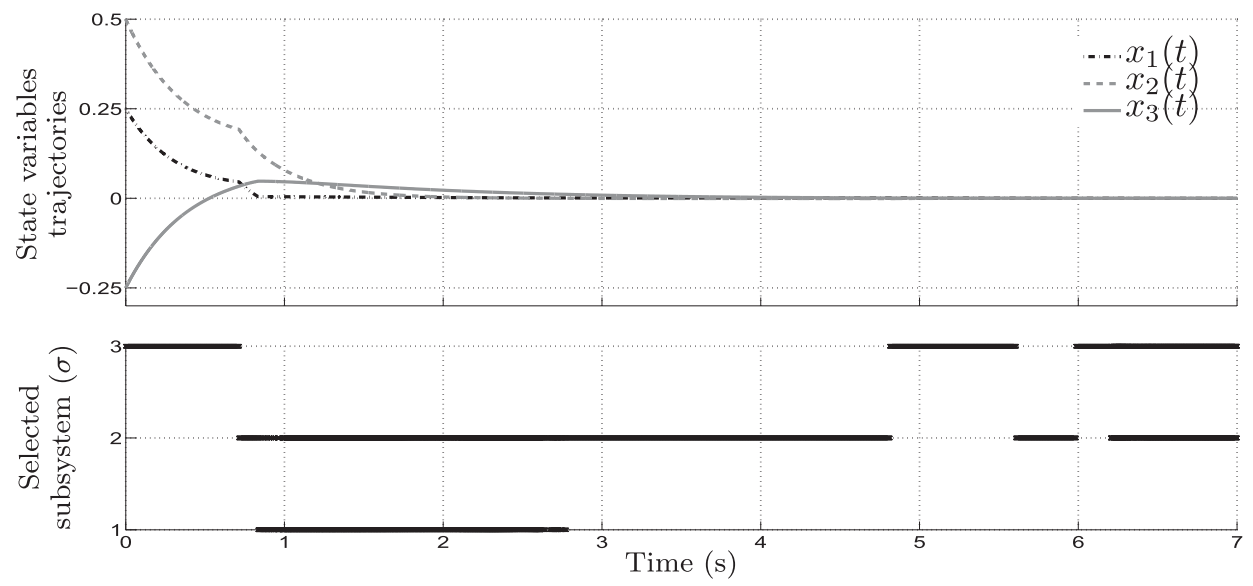

Figure 5. Time response of the state variables and switching selection of the controlled system (1), (2), (6) and (82).

represented. Note that, if $a<-\frac{1}{3}, b<-4, c<-2$, the matrices $A_{1}(a), A_{2}(b)$ and $A_{3}(c)$ are Hurwitz. Otherwise, if $a \geq-\frac{1}{3}, b \geq$ $-4, c \geq-2$, the matrices $A_{1}(a), A_{2}(b)$ and $A_{3}(c)$ are not Hurwitz. The vertices of polytope were obtained considering $0 \leq$ $a \leq 1,-3 \leq b \leq-1.5,-1.5 \leq c \leq-1$. Initially, consider that $\lambda_{1}=0.3, \lambda_{2}=0.4, \lambda_{3}=0.3, x_{0}=x(0)=\left[\begin{array}{lll}0.25 & 0.5 & -0.25\end{array}\right]^{\prime}$ and $u(t)=0$. From conditions of both Theorems 3.2 and 3.3, the obtained guaranteed cost (7) was 6.022. The next subsection is devoted to apply the concepts of DE-LMI algorithm in order to reduce the guaranteed cost.

\subsubsection{Finding the suboptimal parameters of convex combination}

Note that, the conditions (11), (16) and (17) are BMIs, contain terms as the product of a scalar by a matrix. However, as discussed earlier, the proposed hybrid metaheuristic DE-LMI algorithm is able to find feasible solutions in order to reduce the guaranteed cost (7), obtaining values of convex combination parameters that yield suboptimal guaranteed cost value. In this case, the DE-LMI algorithm searches the values of $\lambda_{i}, i \in \mathbb{K}_{N}$, such that, $\sum_{i=1}^{N} \lambda_{i}=1$ and $Q_{\lambda}=\lambda_{1} Q_{1}+\lambda_{2} Q_{2}+\cdots+\lambda_{N} Q_{N}$. From the conditions of Theorem 3.3, the DE-LMI algorithm stopped in the 17th generation due tolerance stop criterion, yielding a value of 2.087 for the guaranteed cost with the solution $\lambda_{1}=0.2449$; $\lambda_{2}=0.3623$; , and $\lambda_{3}=0.3928$; The result shows that the guaranteed cost was considerably reduced when compared with the value obtained disregarding optimisation. Figure 5 presents the trajectories of the state variables and the selected subsystem over time, obtained through of conditions proposed in Theorem 3.3. It is important to highlight that the switching strategy (6) stabilises the system described in (82), even when the state matrices are not Hurwitz. Furthermore, note that when $t>4 \mathrm{~s}$, the time responses of the state variables are close to zero. However, observe that the switching 
strategy continues selecting the best available subsystem considering (6).

\subsection{Example III - numerical simulation}

This numerical example was introduced in Chang et al. (2015). It is represented by (1) and (2), with $r=2, N=1$ (Corollary 4.5) and the following matrices:

The parameter $\delta$ was added in the matrices $A_{1}$ and $A_{2}$ to draw a comparison between Theorem 4.2 (Chang et al., 2015) and Theorem 4.3 for the $\mathscr{H}_{\infty}$ performance, considering $\delta$ increments.

Figure 6 shows that Theorem 4.3 achieves better $\mathscr{H}_{\infty}$ performance when compared with the Theorem 4.2. Furthermore, it

$$
\begin{aligned}
A_{1} & =\left[\begin{array}{ccc}
-0.9896 & 17.41 & 96.15 \\
0.2648 & -0.8512 & -11.39 \\
0 & 0 & -30+\delta
\end{array}\right], \\
A_{2} & =\left[\begin{array}{ccc}
-1.702 & 50.72 & 263.5 \\
0.2201 & -1.418 & -31.99 \\
0 & 0 & -30+\delta
\end{array}\right], \\
B_{1} & =\left[\begin{array}{c}
-97.78 \\
0 \\
30
\end{array}\right], \quad B_{1}=\left[\begin{array}{c}
-85.09 \\
0 \\
30
\end{array}\right], \quad E_{1}=E_{2}=\left[\begin{array}{l}
0 \\
1 \\
1
\end{array}\right], \\
C_{11} & =C_{12}=I_{3 \times 3}, \\
D_{1} & =D_{2}=0, \quad F_{1}=F_{2}=0, \quad C_{21}=\left[\begin{array}{lll}
1 & 0 & 0 \\
0 & 1 & 0
\end{array}\right], \\
C_{21} & =\left[\begin{array}{lll}
1 & 0 & 0 \\
0 & 1 & 1
\end{array}\right], \\
H_{1} & =H_{2}=0 .
\end{aligned}
$$

to highlight that for $\delta>4.9$ the conditions of Theorem 4.2 are unfeasible.

\subsection{Example IV - practical application: semi-active switched suspension}

In order to evaluate the proposed technique presented in the Theorem 4.3 in a practical application, consider the semi-active switched suspension addressed in Geromel et al. (2008) and Cardim et al. (2016). The problem consists in designing an output feedback controller that jointly with the switching strategy mitigates the passenger's discomfort. Figure 7 depicts the system. The mathematical model can be seen as the system represented in (1) and (2) with the following matrices:

$$
A_{1}(\alpha)=\left[\begin{array}{cccc}
0 & 1 & 0 & 0 \\
\frac{-k(\alpha)}{M(\alpha)} & \frac{-c_{\min }}{M(\alpha)} & \frac{k(\alpha)}{M(\alpha)} & \frac{c_{\min }}{M(\alpha)} \\
0 & 0 & 0 & 1 \\
\frac{k(\alpha)}{m} & \frac{c_{\min }}{m} & \frac{-\left(k(\alpha)+k_{t}\right)}{m} & \frac{-c_{\min }}{m}
\end{array}\right],
$$

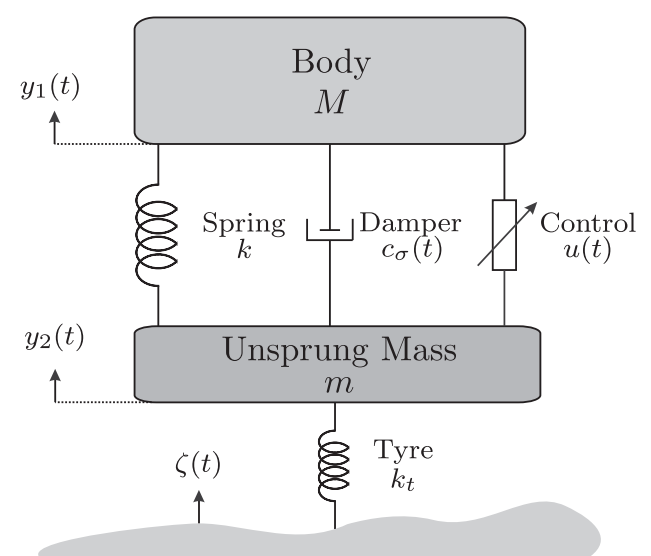

Figure 7. Active suspension system (quarter car).

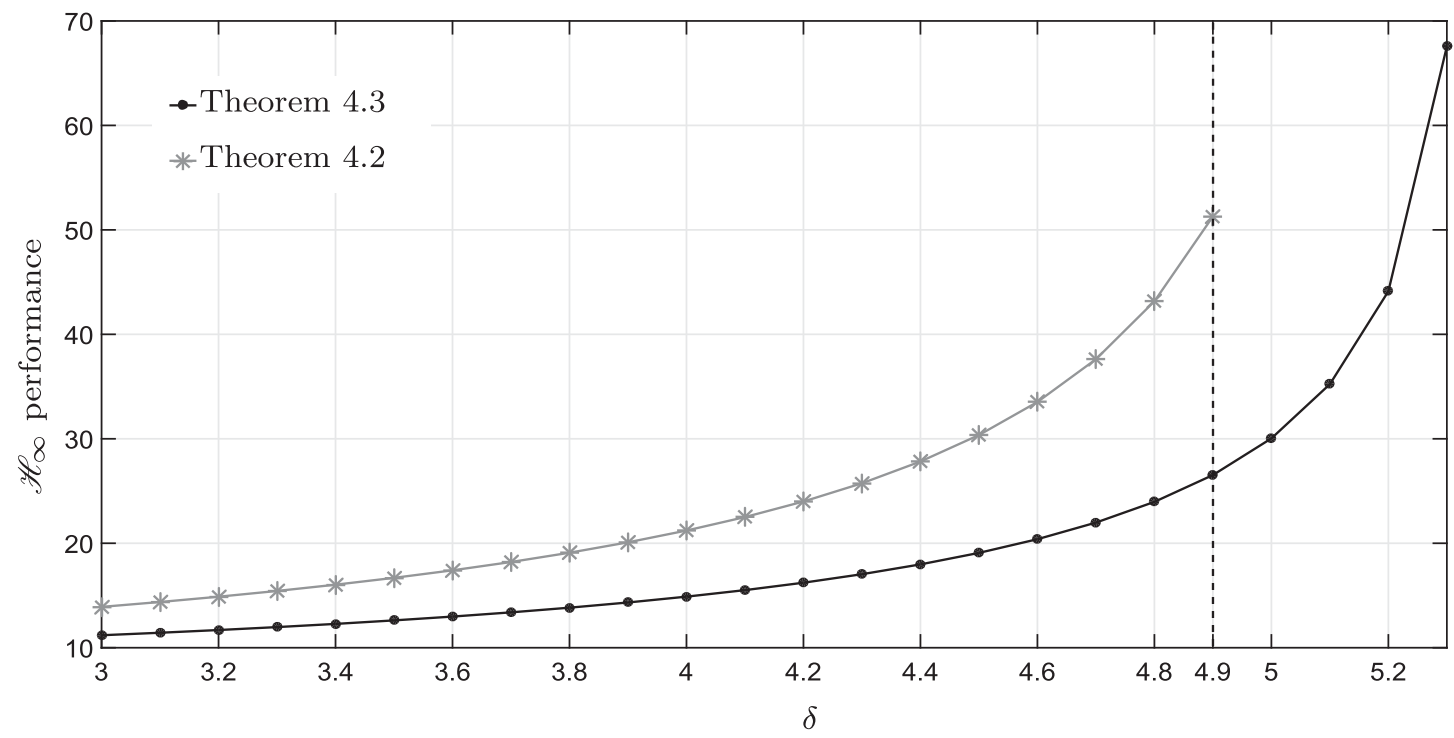

Figure 6. $\mathscr{H}_{\infty}$ cost comparison between Theorems 4.2 and 4.3 for $\delta$ increments. 


$$
\begin{aligned}
& B_{1}(\alpha)=B_{2}(\alpha)=\left[\begin{array}{c}
0 \\
\frac{1}{M(\alpha)} \\
0 \\
\frac{-1}{m}
\end{array}\right] \\
& A_{2}(\alpha)=\left[\begin{array}{cccc}
0 & 1 & 0 & 0 \\
\frac{-k(\alpha)}{M(\alpha)} & \frac{-c_{\max }}{M(\alpha)} & \frac{k(\alpha)}{M(\alpha)} & \frac{c_{\max }}{M(\alpha)} \\
0 & 0 & 0 & 1 \\
\frac{k(\alpha)}{m} & \frac{c_{\max }}{m} & \frac{-\left(k(\alpha)+k_{t}\right)}{m} & \frac{-c_{\max }}{m}
\end{array}\right] \text {, } \\
& E_{1}(\alpha)=E_{2}(\alpha)=\left[\begin{array}{c}
0 \\
0 \\
0 \\
\frac{k_{t}}{m}
\end{array}\right] \text {, } \\
& C_{11}(\alpha)=\left[\begin{array}{llll}
\frac{-k}{M(\alpha)} & \frac{-c_{\min }}{M(\alpha)} & \frac{k}{M(\alpha)} & \frac{c_{\min }}{M(\alpha)}
\end{array}\right] \text {, } \\
& C_{12}(\alpha)=\left[\begin{array}{llll}
\frac{-k}{M(\alpha)} & \frac{-c_{\max }}{M(\alpha)} & \frac{k}{M(\alpha)} & \frac{c_{\max }}{M(\alpha)}
\end{array}\right], \\
& D_{11}=D_{12}=D_{21}=D_{22}=0 \text {, } \\
& F_{11}=F_{12}=F_{21}=F_{22}=0 \\
& C_{21}=C_{22}=\left[\begin{array}{cccc}
1 & 0 & -1 & 0 \\
0 & 1 & 0 & -1
\end{array}\right], \quad H_{1}=H_{2}=0 \text {. }
\end{aligned}
$$

where the state vector is $x(t)=\left[\delta y_{1} \delta \dot{y}_{1} \delta y_{2} \delta \dot{y}_{2}\right], \delta y_{1}$ and $\delta y_{2}$ are the variations of $y_{1}$ and $y_{2}$ around an equilibrium point. Additionally $y_{1}(t), y_{2}(t), \zeta(t)$ are the vertical position of the body, the unsprung mass, and the road profile, respectively. The others plant parameters are the quarter-car body mass $(M)$, the unsprung mass $(m)$, the stiffness of the suspension spring $(k)$, the stiffness of the tire $\left(k_{t}\right)$ and damping coefficient of the passive shock absorber $\left(c_{\sigma}\right)$. Furthermore, it is considered a switching strategy such that the coefficient of the passive shock absorber $\left(c_{\sigma}\right)$ can assume only two values, previously named, $c_{\min }$ and $c_{\max }$. Moreover, it is important to stress that $z(t)$ corresponds to the body acceleration, that is, $\ddot{y}_{1}(t)$.

\subsubsection{Case l: uncertain quarter-car body mass (Cardim et al., 2016)}

For the first simulation it is assumed that the quarter-car body mass is uncertain and belongs to $M_{\min }<M<M_{\max }$. Thus, for this case, the system is represented by (1), (2) and (84) with $r=2, N=2, i \in\{1,2\}, j \in\{1,2\}$. The following values were adopted: $M_{\min }=350 \mathrm{~kg}, M_{\max }=450 \mathrm{~kg}, c_{\min }=$ $3 \times 10^{2}(\mathrm{~N} . \mathrm{s}) / \mathrm{m}, c_{\max }=3.9 \times 10^{3}(\mathrm{~N} . \mathrm{s}) / \mathrm{m}, m=50 \mathrm{~kg}, k=2 \times$ $10^{4} \mathrm{~N} / \mathrm{m}, k_{t}=2.5 \times 10^{5} \mathrm{~N} / \mathrm{m}$. Due to practical implementation issues, the output feedback gains must be bounded. In this sense, through the DE-LMI it is possible to constraint the norm of vectors gains. For this case the values were constrained according to $\operatorname{norm}\left(K_{i s}\right) \leq 10 \times 10^{3}$. The DE-LMI algorithm, considering the solver LMIlab (Gahinet et al., 1994), was applied in order to obtain the output feedback gains $\left(K_{i s}\right)$ and the switching decision matrices $\left(\mathcal{Z}_{i s}\right)$ for the conditions proposed in Theorem 4.3.
The obtained values are the following:

$$
\begin{array}{ll}
\beta=0.1709, & \rho=1.8066 \\
\lambda_{1} \approx 0, & \lambda_{2} \approx 1 \\
K_{11}=\left[\begin{array}{ll}
619.8018 & -0.6575
\end{array}\right], & K_{21}=1 \times 10^{3}\left[\begin{array}{ll}
-7.5318 & -1.0005
\end{array}\right], \\
K_{12}=\left[\begin{array}{ll}
635.0843 & -0.0200
\end{array}\right], & K_{22}=1 \times 10^{3}\left[\begin{array}{ll}
-9.8440 & -1.0180
\end{array}\right], \\
\mathcal{Z}_{11}=1 \times 10^{7}\left[\begin{array}{cc}
-0.5164 & -1.7135 \\
-1.7135 & 0.0129
\end{array}\right], & \mathcal{Z}_{12}=1 \times 10^{7}\left[\begin{array}{cc}
-0.5163 & -1.7138 \\
-1.7138 & 0.0261
\end{array}\right], \\
\mathcal{Z}_{21}=1 \times 10^{7}\left[\begin{array}{ll}
-0.5164 & -1.7135 \\
-1.7135 & 0.0126
\end{array}\right], & \mathcal{Z}_{22}=1 \times 10^{7}\left[\begin{array}{cc}
-0.5164 & -1.7135 \\
-1.7135 & 0.0126
\end{array}\right],
\end{array}
$$

The road profile is treated in this paper as the exogenous disturbance, that is $w(t)=\zeta(t)$. It is important to highlight that in Cardim et al. (2016) the guaranteed cost was selected as the performance criterion and there is no $\mathscr{H}_{\infty}$ approach involved. For comparison purpose with the simulation results presented in Cardim et al. (2016), the same road profile $(w(t)=\zeta(t))$, a square wave with amplitude $\pm 4 \mathrm{~cm}$, was considered. Since the quarter-car body mass is an uncertain parameter $(M)$, the simulation examines two different scenarios, for $M=M_{\min }=$ $350 \mathrm{~kg}(0 \leq t<4 \mathrm{~s})$ and $M=M_{\max }=450 \mathrm{~kg}(t>4 \mathrm{~s})$. For the open-loop simulation, it was used the maximum value of $c_{\sigma}$, in this case $c_{\max }=3.9 \times 10^{3}(\mathrm{~N} . \mathrm{s}) / \mathrm{m}$. Figures 8,9 and 10 show the simulation results. Figure 8 shows the comparison of the time response of $y_{1}(t)$ and $y_{2}(t)$ for the conditions of Theorem 4.3, the conditions introduced in Cardim et al. (2016) in Section 5.2 , and the uncontrolled situation with $c_{\max }$. It is possible to observe that the proposed Theorem 4.3 presents the best performance for $y_{1}(t)$, considering the reduction of the guaranteed cost. This fact is linked with the reduction of $\int_{0}^{t} z(t)^{\prime} z(t) \mathrm{d} t$, as depicted in Figure 9, since $z(t)$ corresponds to the body acceleration $\ddot{y}_{1}(t)$. Theorem 4.3 provides about $20.4 \%$ and $7.11 \%$ of reduction in the $\int_{0}^{t} z(t)^{\prime} z(t) \mathrm{d} t$ final value, when compared to the open-loop case and the response related to the conditions proposed in Cardim et al. (2016), respectively. It is important to stress that the proposed DE-LMI method do not ensure the global optimisation, due to non-convex characteristics related to BMIs. Therefore different values for the controllers can be found, depending on the ED initialisation parameters values (Storn \& Price, 1997). Figure 10 shows the control input $u(t)$ time response. The switching selection was omitted in this example, as in this case for Theorem 4.3 the switching function returned $\sigma=2$ and $\eta=1$ for the simulation time, that is, the controller gain $K_{21}$ was kept. Concerning the dynamical output feedback design proposed in Geromel et al. (2008), note that it can not be directly applied in this example that presents uncertainties since in the aforementioned paper the plant was supposed known and without uncertain parameters.

\subsubsection{Case II: uncertain quarter-car body mass (M) and suspension spring stiffness $(k)$ variation}

For this second case besides the quarter-car body mass the stiffness of the suspension spring was treated as an uncertain parameters, belonging to $k_{\min }<k<k_{\max }$. Now, the system is represented by (1), (2) and (84) with $r=4, N=2, i \in\{1,2\}$, $j \in\{1,2,3,4\}$. The following values were adopted: $M_{\min }=$ $300 \mathrm{~kg}, M_{\max }=500 \mathrm{~kg}, c_{\min }=6 \times 10^{2}(\mathrm{~N} . \mathrm{s}) / \mathrm{m}, c_{\max }=9 \times$ $10^{2}(\mathrm{~N} . \mathrm{s}) / \mathrm{m}, \quad m=50 \mathrm{~kg}, \quad k_{\min }=2 \times 10^{4} \mathrm{~N} / \mathrm{m}, \quad k_{\max }=12 \times$ $10^{4} \mathrm{~N} / \mathrm{m}, k_{t}=2.5 \times 10^{5} \mathrm{~N} / \mathrm{m}$. It is important to highlight that 


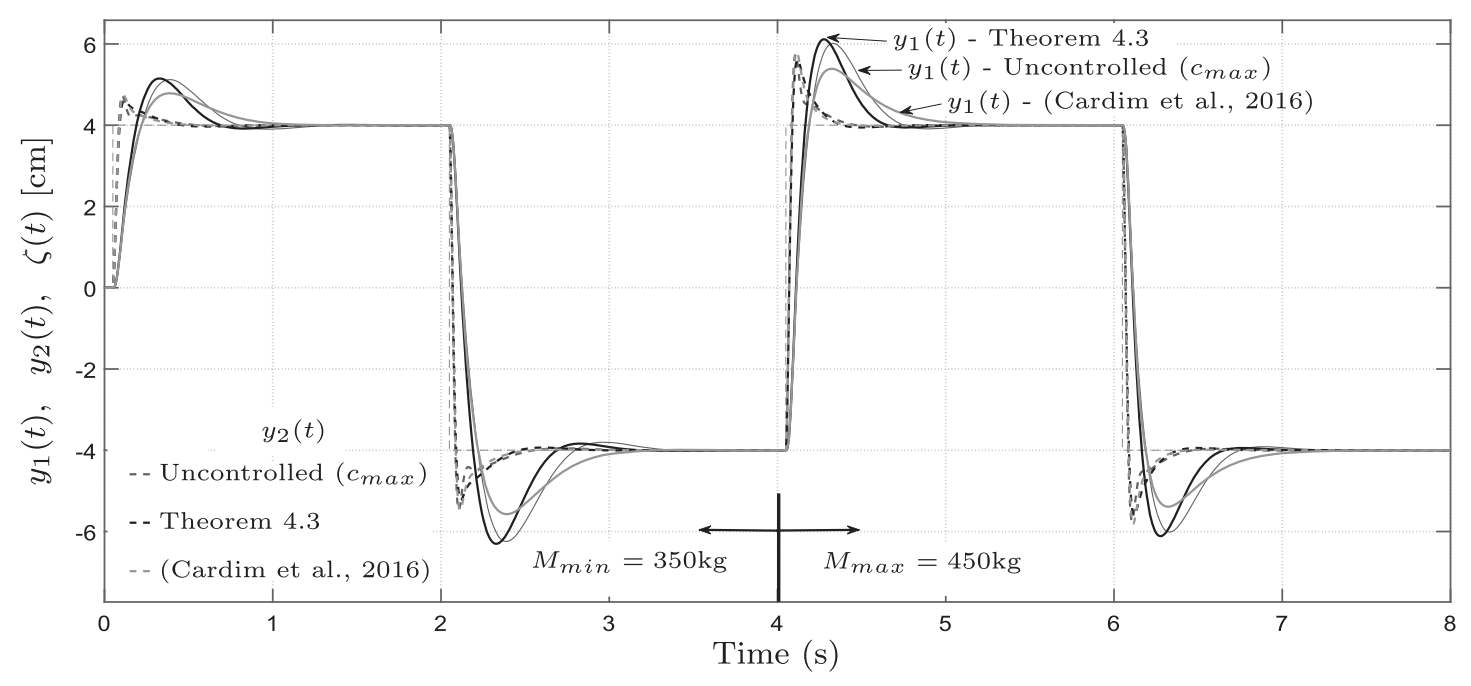

Figure 8. Comparison of the time response: $M=M_{\min }=350 \mathrm{~kg}(0 \leq t<4 \mathrm{~s})$ and $M=M_{\max }=450 \mathrm{~kg}(t>4 \mathrm{~s})$.

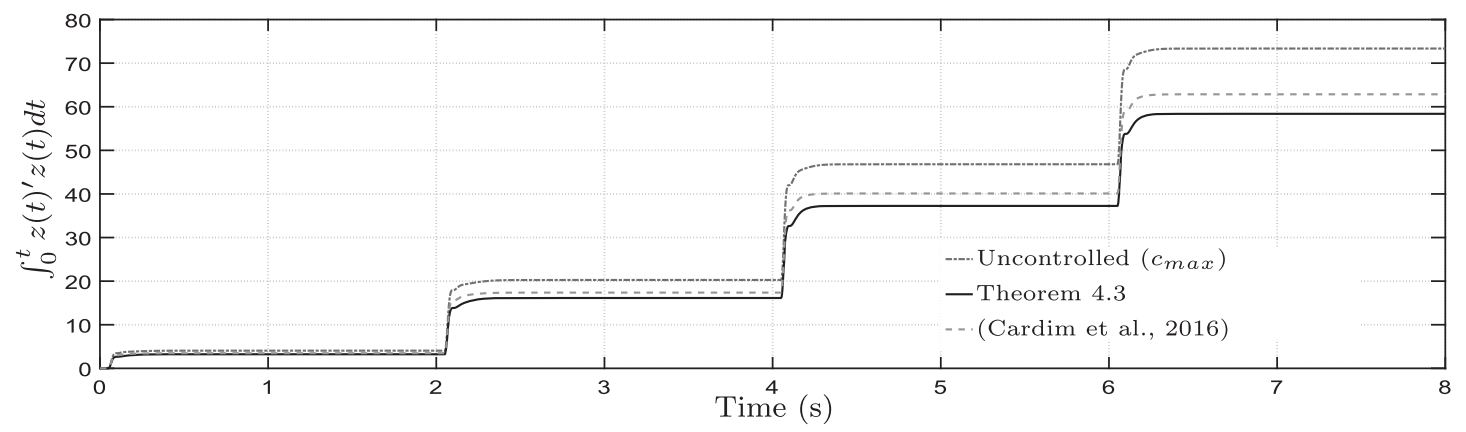

Figure 9. Comparison of the guaranteed cost: $M=M_{\min }=350 \mathrm{~kg}(0 \leq t<4 \mathrm{~s})$ and $M=M_{\max }=450 \mathrm{~kg}(t>4 \mathrm{~s})$.

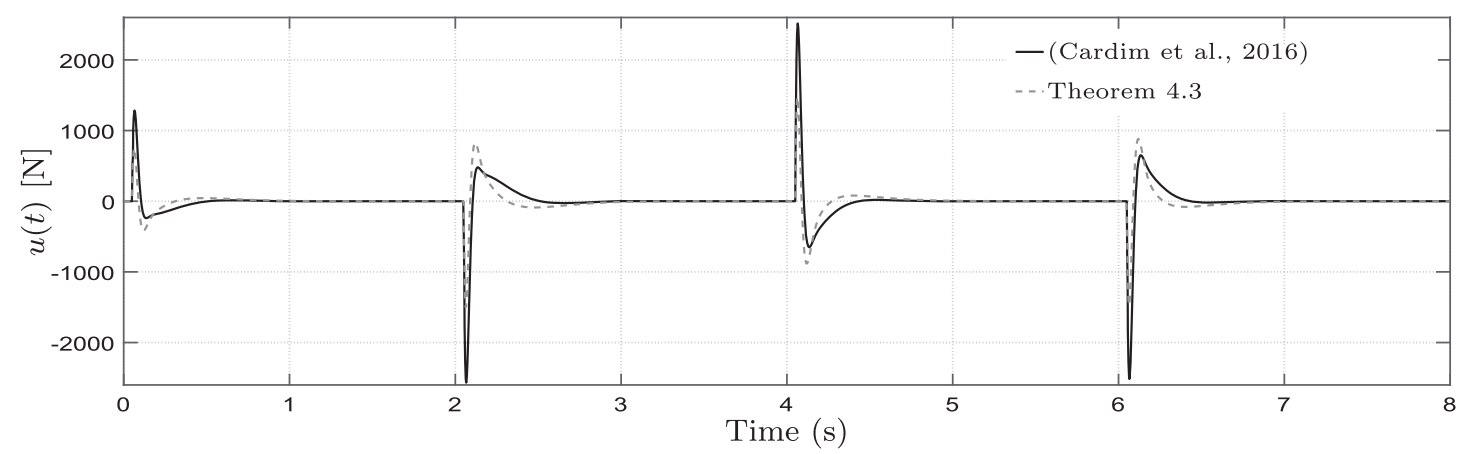

Figure 10. Control signal $u(t): M=M_{\min }=350 \mathrm{~kg}(0 \leq t<4 \mathrm{~s})$ and $M=M_{\max }=450 \mathrm{~kg}(t>4 \mathrm{~s})$.

the range of the quarter-car body mass was increased and the suspension spring stiffness was treated as another uncertainty parameter with the purpose of exploiting and to show the potential of Theorem 4.3. For this case, the norm of the vectors gains was also constrained according to norm $\left(K_{i s}\right) \leq 10 \times 10^{3}$. The DE-LMI algorithm, considering the solver LMIlab (Gahinet et al., 1994), was used for the conditions proposed in Theorem 4.3. The obtained values for the parameters $\lambda_{i}, \rho, \beta$, for the controller gains $K_{i s}$ and the switching decision matrices $\mathcal{Z}_{i s}$ are the following:

$$
\begin{aligned}
\beta & =0.2559, \\
\lambda_{1} & =0.15020,
\end{aligned}
$$

$$
\begin{aligned}
& K_{11}=1 \times 10^{3}\left[\begin{array}{ll}
-7.1936 & -0.7666
\end{array}\right] \text {, } \\
& K_{12}=1 \times 10^{3}\left[\begin{array}{ll}
-5.5566 & -0.7633
\end{array}\right] \text {, } \\
& K_{13}=1 \times 10^{3}\left[\begin{array}{ll}
-5.0453 & -0.7935
\end{array}\right] \text {, } \\
& K_{14}=1 \times 10^{3}\left[\begin{array}{ll}
-4.3620 & -0.7681
\end{array}\right] \text {, } \\
& \mathcal{Z}_{11}=\left[\begin{array}{ll}
30444329.2000 & 26631481.8465 \\
26631481.8465 & 5045455.02273
\end{array}\right] \text {, } \\
& \mathcal{Z}_{12}=\left[\begin{array}{ll}
30444329.2000 & 26631481.8469 \\
26631481.8469 & 5045455.01505
\end{array}\right] \text {, } \\
& \mathcal{Z}_{13}=\left[\begin{array}{ll}
30444329.1998 & 26631481.8478 \\
26631481.8478 & 5045455.00590
\end{array}\right] \text {, }
\end{aligned}
$$




$$
\begin{aligned}
& \mathcal{Z}_{14}=\left[\begin{array}{ll}
30444329.1999 & 26631481.8477 \\
26631481.8477 & 5045455.00677
\end{array}\right], \\
& \rho=-0.3026 \\
& \lambda_{2}=0.8498 \\
& K_{21}=1 \times 10^{3}\left[\begin{array}{ll}
-9.3346 & -0.9770
\end{array}\right] \text {, } \\
& K_{22}=1 \times 10^{3}\left[\begin{array}{ll}
-7.4555 & -0.9792
\end{array}\right] \text {, } \\
& K_{23}=1 \times 10^{3}\left[\begin{array}{ll}
-7.2094 & -0.9824
\end{array}\right] \text {, } \\
& K_{24}=1 \times 10^{3}\left[\begin{array}{ll}
-6.4612 & -0.9838
\end{array}\right] \text {, }
\end{aligned}
$$$$
\mathcal{Z}_{21}=\left[\begin{array}{ll}
30444329.1999 & 26631481.8476 \\
26631481.8476 & 5045454.97914
\end{array}\right]
$$
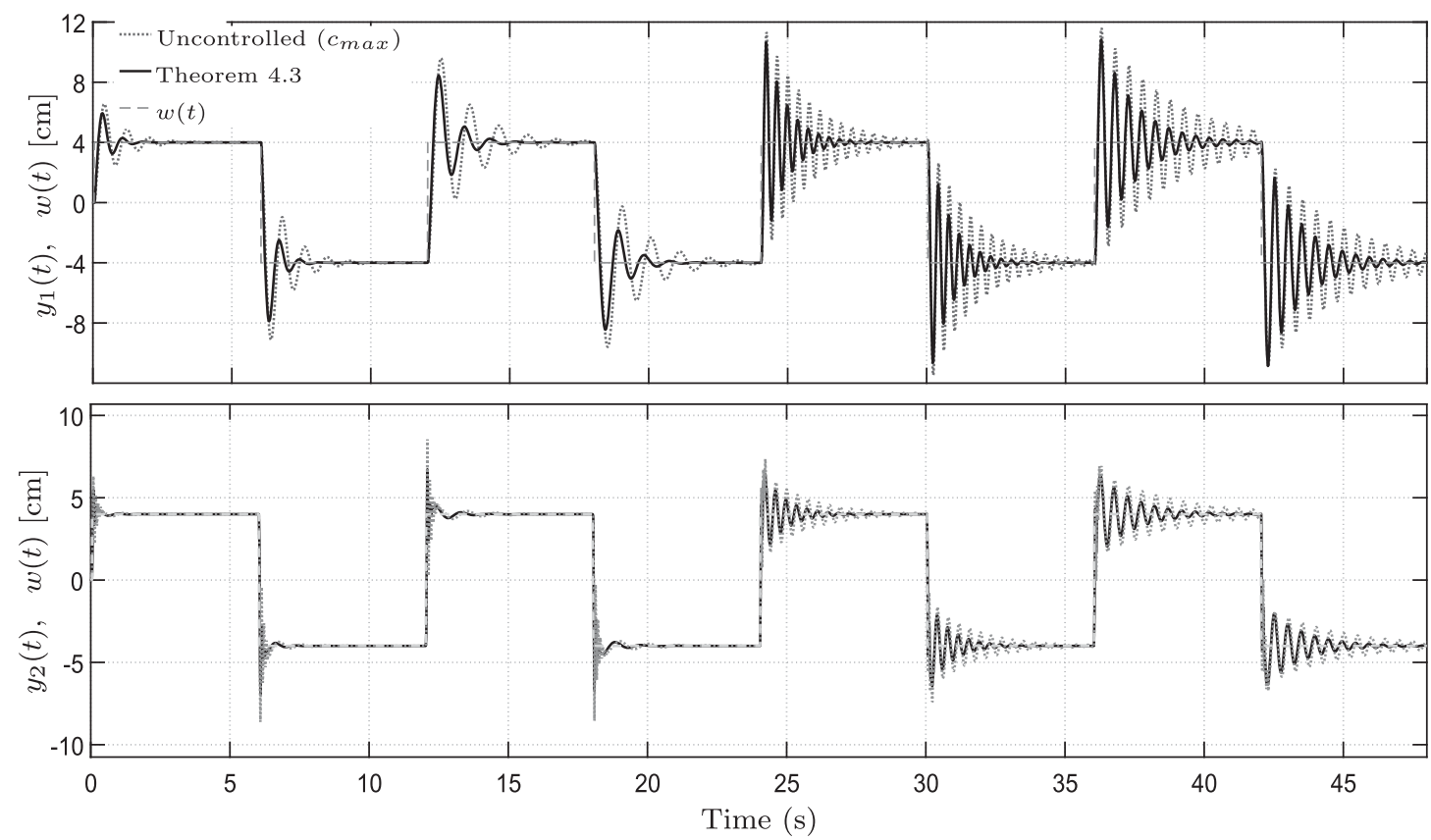

Figure 11. Comparison of the time response: $M=M_{\min }=300 \mathrm{~kg}$ and $k=k_{\min }=2 \times 10^{4} \quad(0 \leq t<12 \mathrm{~s}), M=M_{\max }=500 \mathrm{~kg}$ and $k=k_{\min }=2 \times 10^{4}$ $(12 \leq t<24 \mathrm{~s}), M=M_{\min }=300 \mathrm{~kg}$ and $k=k_{\max }=12 \times 10^{4}(24 \leq t<36 \mathrm{~s})$, and $M=M_{\max }=500 \mathrm{~kg}$ and $k=k_{\max }=12 \times 10^{4}(36 \leq t<48 \mathrm{~s})$.
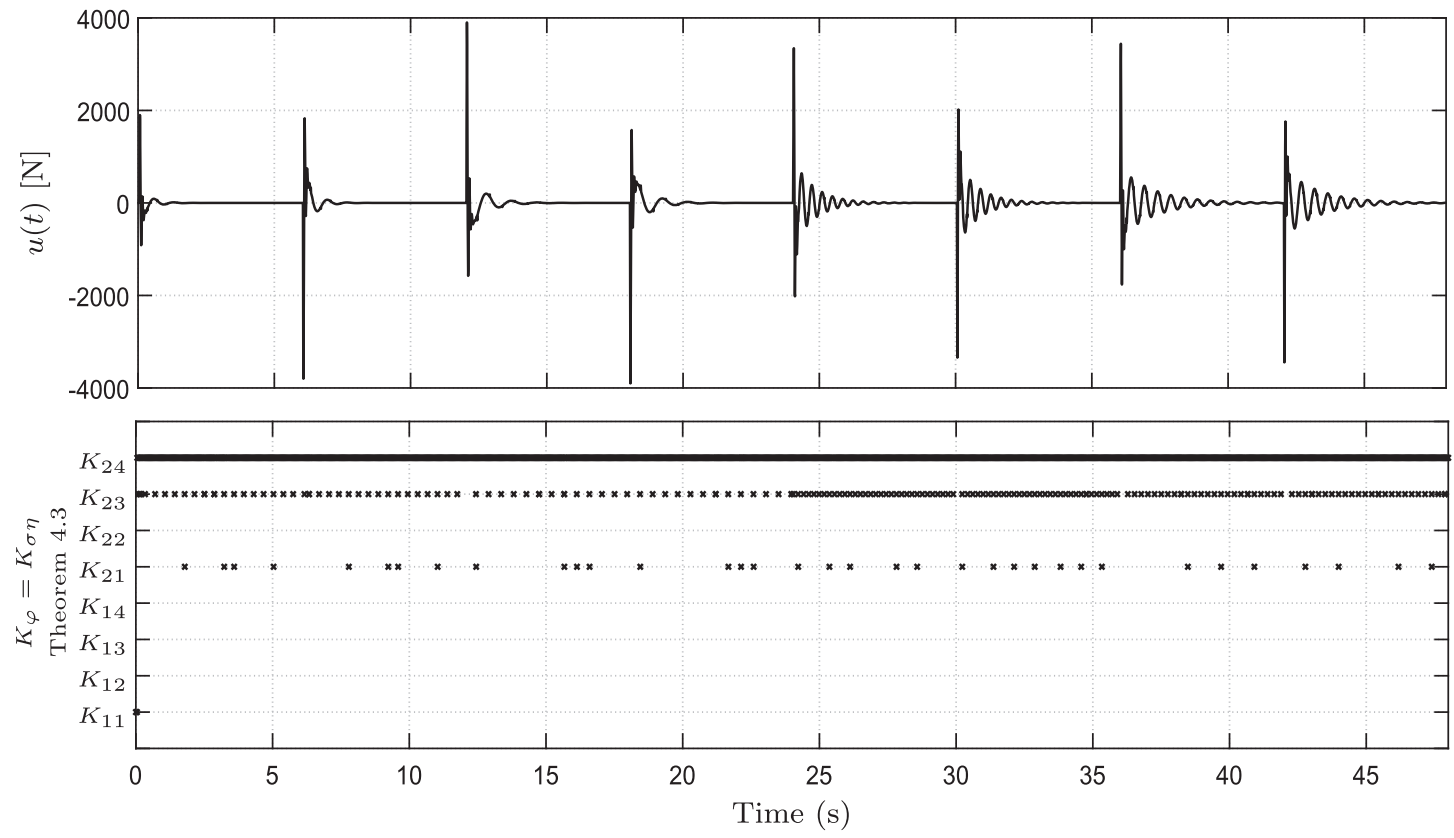

Figure 12. Control signal $u(t)$ and switching selection: $M=M_{\min }=300 \mathrm{~kg}$ and $k=k_{\min }=2 \times 10^{4}(0 \leq t<12 \mathrm{~s}), M=M_{\max }=500 \mathrm{~kg}$ and $k=k_{\min }=2 \times 10^{4}$ $(12 \leq t<24 \mathrm{~s}), M=M_{\min }=300 \mathrm{~kg}$ and $k=k_{\max }=12 \times 10^{4}(24 \leq t<36 \mathrm{~s})$, and $M=M_{\max }=500 \mathrm{~kg}$ and $k=k_{\max }=12 \times 10^{4}(36 \leq t<48 \mathrm{~s})$. 
For the open-loop simulation, it was used the maximum value of $c_{\sigma}$, in this case $c_{\max }=9 \times 10^{2}(\mathrm{~N} . \mathrm{s}) / \mathrm{m}$. The simulation examines four different scenarios: (1) $M=M_{\min }=300 \mathrm{~kg}$ and $k=k_{\min }=2 \times 10^{4} \mathrm{~N} / \mathrm{m}(0 \leq t<12 \mathrm{~s})$, (2) $M=M_{\max }=$ $500 \mathrm{~kg}$ and $k=k_{\min }=2 \times 10^{4} \mathrm{~N} / \mathrm{m}(12 \leq t<24 \mathrm{~s})$, (3) $M=$ $M_{\min }=300 \mathrm{~kg}$ and $k=k_{\max }=12 \times 10^{4} \mathrm{~N} / \mathrm{m}(24 \leq t<36 \mathrm{~s})$ and (4) $M=M_{\max }=300 \mathrm{~kg}$ and $k=k_{\max }=12 \times 10^{4} \mathrm{~N} / \mathrm{m}$ $(t>36 \mathrm{~s})$. Figure 11 shows the comparison of the time response of $y_{1}(t)$ and $y_{2}(t)$ for the conditions of Theorem 4.3 and the open-loop system. It is possible to observe that the oscillation and peak values of $y_{1}(t)$ and $y_{2}(t)$ are significantly reduced when considering the switching controllers. Finally, Figure 12 shows the control input signal $(u(t))$ and the controllers/subsystems switching.

\section{Conclusions}

Initially, this paper proposed in Theorem 3.3 a strategy to design an exclusive output-dependent switching strategy for controlling linear time-invariant continuous-time uncertain switched linear systems.

A proof in Theorem 3.4 shows that, if the known conditions of Theorem 3.2 hold, then the conditions proposed in Theorem 3.3 also hold. Furthermore, from simulations results (Examples I and II), the conditions proposed in Theorem 3.3 present a greater feasible region and reduce the guaranteed cost when compared with the conditions of Theorem 3.2. Therefore, the conditions proposed in Theorem 3.3 are less conservative than that presented in Theorem 3.2. The second control problem studied in this paper was the robust switching SOF $\mathscr{H}_{\infty}$ control of continuous-time switched linear time-invariant systems. For a particular case of switched systems with only one subsystem, a proof in Theorem 4.4 shows that if the known conditions of Theorem 4.2 hold, then the conditions proposed in Theorem 4.3 also hold.

Additionally, from simulations results (Example III), the conditions proposed in Theorem 4.3 present a greater feasible region and reduce the $\mathscr{H}_{\infty}$ cost when compared with the conditions of Theorem 4.2. Therefore, the conditions proposed in Theorem 4.3 are less conservative than that presented in Theorem 4.2 .

The conditions of the proposed methods are a special class of BMIs, which contain some bilinear terms as the product of a matrix and a scalar, related to a suitable convex combination and two scalar parameters to provide extra free dimensions in the solution space. The hybrid algorithm DE-LMI, is proposed for obtaining feasible solutions of this particular NP-hard problem.

Finally, in Example IV, it was presented a practical application on a semi-active suspension system. It was possible to observe a dynamic response improvement considering the reduction of the guaranteed cost when compared with the results obtained considering the procedure presented in Cardim et al. (2016). The second study regarding this problem, considering an uncertain bounded mass and a fault in the spring, confirms the effectiveness of the proposed approach. Future research on this subject is to extend the proposed methodology for designing switched dynamic output feedback controllers for the same class of uncertain switched linear systems studied in this paper.

\section{Disclosure statement}

No potential conflict of interest was reported by the authors.

\section{Funding}

This work was supported by Conselho Nacional de Desenvolvimento Científico e Tecnológico [310798/2014-0 and 300703/2013-9], Coordenação de Aperfeiçoamento de Pessoal de Nível Superior (CAPES) and Fundação de Amparo à Pesquisa do Estado de São Paulo [2011/17610-0].

\section{ORCID}

Leonardo Ataide Carniato (D) http://orcid.org/0000-0003-3481-3694 Alexandre Ataide Carniato (D) http://orcid.org/0000-0001-7009-6624 Marcelo Carvalho Minhoto Teixeira (D) http://orcid.org/0000-0002-29962831

Rodrigo Cardim (D) http://orcid.org/0000-0002-1072-3814

Edson Italo Mainardi Junior (D) http://orcid.org/0000-0002-8526-5643

Edvaldo Assunção (iD) http://orcid.org/0000-0002-4439-8570

\section{References}

Agulhari, C. M., Oliveira, R. C. L. F., \& Peres, P. L. D. (2010). Static output feedback control of polytopic systems using polynomial Lyapunov functions. 49th IEEE conference on decision and control (CDC), Atlanta, GA, USA, 15-17 December 2010 (pp. 6894-6901).

Ban, J., Kwon, W., Won, S., \& Kim, S. (2018). Robust $\mathscr{H}_{\infty}$ finitetime control for discrete-time polytopic uncertain switched linear systems. Nonlinear Analysis: Hybrid Systems, 29, 348-362. Retrieved from http://www.sciencedirect.com/science/article/pii/S1751570X18300244

Boyd, S., ElGhaoui, L., Feron, E., \& Balakrishnan, V. (1994). Linear matrix inequalities in system and control theory (Vol. 15). Philadelphia, PA: SIAM.

Cardim, R., Teixeira, M. C. M., Assunção, E., \& Covacic, M. R. (2009). Variable-structure control design of switched systems with an application to a DC-DC power converter. IEEE Transactions on Industrial Electronics, 56(9), 3505-3513.

Cardim, R., Teixeira, M. C. M., Assunção, E., Ribeiro, J. M. S., Covacic, M. R., \& Gaino, R. (2016). Robust switched control based on strictly positive real systems and variable structure control techniques. International Journal of Adaptive Control and Signal Processing, 30(8-10), 1244-1268.

Chang, X. H., Park, J. H., \& Zhou, J. (2015). Robust static output feedback $\mathscr{H}_{\infty}$ control design for linear systems with polytopic uncertainties. Systems and Control Letters, 85, 23-32. Retrieved from doi:10.1016/j.sysconle.2015.08.007

Crusius, C. A. R., \& Trofino, A. (1999). Sufficient LMI conditions for output feedback control problems. IEEE Transactions on Automatic Control, 44(5), 1053-1057.

Daafouz, J., Riedinger, P., \& Iung, C. (2002, November). Stability analysis and control synthesis for switched systems: A switched lyapunov function approach. IEEE Transactions on Automatic Control, 47(11), $1883-1887$.

Das, S., \& Suganthan, P. N. (2011 February). Differential evolution: A survey of the state-of-the-art. IEEE Transactions on Evolutionary Computation, 15(1), 4-31

Deaecto, G. S., Geromel, J. C., \& Daafouz, J. (2011). Switched state-feedback control for continuous time-varying polytopic systems. International Journal of Control, 84(9), 1500-1508.

Deaecto, G. S., Geromel, J. C., Garcia, F. S., \& Pomilio, J. A. (2010). Switched affine systems control design with application to DC-DC converters. IET Control Theory \& Applications, 4(7), 1201-1210.

de Souza, W. A., Teixeira, M. C. M., Cardim, R., \& Assunção, E. (2014, December). On switched regulator design of uncertain nonlinear systems using Takagi-Sugeno fuzzy models. IEEE Transactions on Fuzzy Systems, 22(6), 1720-1727.

Ding, D. W., \& Yang, G. H. (2009). Static output feedback control for discrete-time piecewise linear systems: An LMI approach. Acta Automatica Sinica, 35(4), 337-344. 
Dong, J., \& Yang, G. H. (2013). Robust static output feedback control synthesis for linear continuous systems with polytopic uncertainties. Automatica, 49(6), 1821-1829.

Gahinet, P., Nemirovskii, A., Laub, A. J., \& Chilali, M. (1994). The LMI control toolbox. Proceedings of 1994 33rd IEEE Conference on Decision and Control, Lake Buena Vista, FL, 1994 (Vol. 3, pp. 2038-2041).

Geromel, J., Colaneri, P., \& Bolzern, P. (2008). Dynamic output feedback control of switched linear systems. IEEE Transactions on Automatic Control, 53(3), 720-733.

Koumboulis, F. N., \& Tzamtzi, M. P. (2007). A metaheuristic approach for controller design of multivariable processes. 2007 IEEE Conference on Emerging Technologies and Factory Automation (EFTA 2007), Patras, Greece, 25-28 September 2007 (pp. 1429-1432).

Liberzon, D. (2003). Switching in systems and control. Boston: Birkhauser.

Lin, H., \& Antsaklis, P. J. (2007). Switching stabilizability for continuoustime uncertain switched linear systems. IEEE Transactions on Automatic Control, 52(4), 633-646.

Lin, H., \& Antsaklis, P. J. (2009). Stability and stabilizability of switched linear systems: A survey of recent results. IEEE Transactions on Automatic control, 54(2), 308-322.

Liu, J., Vazquez, S., Wu, L., Marquez, A., Gao, H., \& Franquelo, L. G. (2017). Extended state observer-based sliding-mode control for threephase power converters. IEEE Transactions on Industrial Electronics, 64(1), 22-31.

Liu, X., \& Zhang, Q. (2003). Approaches to quadratic stability conditions and $\mathcal{H}_{\infty}$ control designs for T-S fuzzy systems. IEEE Transactions on Fuzzy Systems, 11(6), 830-839.

Mainardi Júnior, E. I., Teixeira, M. C. M., Cardim, R., Assunção, E., M. R. Moreira, de Oliveira, D. R., .. Carniato, A. A. (2015, October 1). Robust control of switched linear systems with output switching strategy. Journal of Control, Automation and Electrical Systems, 26(5), 455-465.

Mozelli, L. A., \& Palhares, R. M. (2011). Stability analysis of linear timevarying systems: Improving conditions by adding more information about parameter variation. Systems \& Control Letters, 60(5), 338-343.

Papageorgiou, M., Diakaki, C., Dinopoulou, V., Kotsialos, A., \& Wang, Y. (2003). Review of road traffic control strategies. Proceedings of the IEEE, 91(12), 2043-2067.

Peaucelle, D., \& Arzelier, D. (2005). Ellipsoidal sets for resilient and robust static output-feedback. IEEE Transactions on Automatic Control, 50(6), 899-904.

Price, K., Storn, R. M., \& Lampinen, J. A. (2006). Differential evolution: A practical approach to global optimization. Berlin: Springer-Verlag.

Qiu, J., Feng, G., \& Yang, J. (2008). Robust mixed $\mathscr{H}_{2} / \mathscr{H}_{\infty}$ filtering design for discrete-time switched polytopic linear systems. IET Control Theory \& Applications, 2(5), 420-430.

Sadabadi, M. S., \& Peaucelle, D. (2016). From static output feedback to structured robust static output feedback: A survey. Annual Reviews in Control, 42, 11-26. Retrieved from doi:10.1016/j.arcontrol.2016.09.014

Sandou, G. (2013). Metaheuristic optimization for the design of automatic control laws. London: ISTE-Wiley.

Shi, S., Wang, S., Ren, S., \& Fei, Z. (2017). Dynamic output feedback $H_{\infty} H_{\infty} \mathscr{H}_{\infty}$ control for continuous-time switched systems. IECON 2017 - 43rd Annual Conference of the IEEE Industrial Electronics Society, Beijing, 2017 (pp. 7529-7534).

Silva, E. R. P., Assunção, E., Teixeira, M. C. M., \& Cardim, R. (2013). Robust controller implementation via state-derivative feedback in an active suspension system subjected to fault. 2013 Conference on control and fault-tolerant systems (SysTol), Nice, France, 9-11 October 2013 (pp. 752-757).

Storn, R., \& Price, K. (1997). Differential evolution - a simple and efficient heuristic for global optimization over continuous spaces. Journal of Global Optimization, 11(4), 341-359.

Sturm, J. F. (1999). Using SeDuMi 1.02, a MATLAB toolbox for optimization over symmetric cones. Optimization methods and software, 11(1-4), 625-653.

Syrmos, V. L., Abdallah, C. T., Dorato, P., \& Grigoriadis, K. (1997). Static output feedback - a survey. Automatica, 33(2), 125-137.

Teixeira, M. C. M., Assunção, E., \& Avellar, R. G. (2003). On relaxed LMI-based designs for fuzzy regulators and fuzzy observers. IEEE Transactions on Fuzzy Systems, 11(5), 613-623.
Wicks, M. A., Peleties, P., \& DeCarlo, R. A. (1994). Construction of piecewise Lyapunov functions for stabilizing switched systems. Proceedings of the 33rd IEEE conference on decision and control, Lake Buena Vista, FL, USA, 14-16 December 1994 (Vol. 4, pp. 3492-3497).

Wu, L., Gao, Y., Liu, J., \& Li, H. (2017). Event-triggered sliding mode control of stochastic systems via output feedback. Automatica, 82, 79-92. Retrieved from doi:10.1016/j.automatica.2017.04.032

Yu, Q., \& Wu, B. (2015). Robust stability analysis of uncertain switched linear systems with unstable subsystems. International Journal of Systems Science, 46(7), 1278-1287.

Yu, Q., \& Zhao, X. (2016). Stability analysis of discrete-time switched linear systems with unstable subsystems. Applied Mathematics and Computation, 273, 718-725.

Zhai, G., Lin, H., \& Antsaklis, P. J. (2003). Quadratic stabilizability of switched linear systems with polytopic uncertainties. International Journal of Control, 76(7), 747-753.

Zhang, W., \& Hu, J. (2008). Dynamic buffer management using optimal control of hybrid systems. Automatica, 44(7), 1831-1840.

Zhang, L., Zhuang, S., \& Braatz, R. D. (2016). Switched model predictive control of switched linear systems: Feasibility, stability and robustness. Automatica, 67, 8-21.

\section{Appendix. Proof of Theorem 3.1}

Consider that (4) and (5) are feasible. It is known that the minimum of a set of real numbers is less than or equal to an arbitrary convex combination of these numbers. Then, from (5) and (6), for $x \neq 0$ it follows that:

$$
0>x^{\prime}\left(Q_{0 j k}+C^{\prime} Q_{\lambda} C\right) x \geq x^{\prime} Q_{0 j k} x+\min _{i \in \mathbb{K}_{N}}\left(y^{\prime} Q_{i} y\right)=x^{\prime}\left(Q_{0 j k}+C^{\prime} Q_{\sigma} C\right) x,
$$

where $\lambda=\left[\lambda_{1} \lambda_{2} \ldots \lambda_{N}\right], \sum_{i=1}^{N} \lambda_{i}=1$ and $\lambda_{i} \geq 0$, for all $i \in \mathbb{K}_{N}$. Observe that $(\mathrm{A} 1)$ can be rewritten as:

$$
\begin{aligned}
& x^{\prime}\left(Q_{0 j k}+C^{\prime} Q_{\sigma} C\right) x \\
& x^{\prime}\left[\begin{array}{c}
I_{n} \\
A(\sigma, \alpha)
\end{array}\right]^{\prime}\left[\begin{array}{cc}
Q_{0 j k}+C^{\prime} Q_{\sigma} C & 0 \\
0 & 0
\end{array}\right]\left[\begin{array}{c}
I_{n} \\
A(\sigma, \alpha)
\end{array}\right] x<0 .
\end{aligned}
$$

Thus, multiplying (A2) by $\alpha_{j} \times \alpha_{k}$ and taking the sum from $j=1$ to $j=r$ and $k=1$ to $k=r$, respectively, from (4), note that:

$$
\begin{aligned}
& 0>\sum_{k=1}^{r} \alpha_{k} \sum_{j=1}^{r} \alpha_{j} x^{\prime}\left[\begin{array}{c}
I_{n} \\
A(\sigma, \alpha)
\end{array}\right]^{\prime} \\
& \times\left[\begin{array}{cc}
Q_{0 j k}+Q_{0 k j}+2 C^{\prime} Q_{\sigma} C & 0 \\
0 & 0
\end{array}\right]\left[\begin{array}{c}
I_{n} \\
A(\sigma, \alpha)
\end{array}\right] x \\
& >\sum_{k=1}^{r} \alpha_{k} \sum_{j=1}^{r} \alpha_{j} x^{\prime}\left[\begin{array}{c}
I_{n} \\
A(\sigma, \alpha)
\end{array}\right]^{\prime} \\
& \times\left[\begin{array}{c}
X_{1 \sigma k} A_{\sigma j}+A_{\sigma j}^{\prime} X_{1 \sigma k}^{\prime}+X_{1 \sigma j} A_{\sigma k}+A_{\sigma k}^{\prime} X_{1 \sigma j}^{\prime} \\
P_{j k}-X_{1 \sigma k}^{\prime}+X_{2 \sigma k} A_{\sigma j}+P_{k j}-X_{1 \sigma j}^{\prime}+X_{2 \sigma j} A_{\sigma j}
\end{array}\right. \\
& \left.\begin{array}{c}
P_{j k}-X_{1 \sigma k}+A_{\sigma j}^{\prime} X_{2 \sigma k}^{\prime}+P_{k j}-X_{1 \sigma j}+A_{\sigma k}^{\prime} X_{2 \sigma j}^{\prime} \\
-X_{2 \sigma k}-X_{2 \sigma k}^{\prime}-X_{2 \sigma j}-X_{2 \sigma j}^{\prime}
\end{array}\right]\left[\begin{array}{c}
I_{n} \\
A(\sigma, \alpha)
\end{array}\right] x \\
& =2 \sum_{k=1}^{r} \alpha_{k} \sum_{j=1}^{r} \alpha_{j} x^{\prime}\left[\begin{array}{c}
I_{n} \\
A(\sigma, \alpha)
\end{array}\right]^{\prime} \\
& \times\left[\begin{array}{cc}
X_{1 \sigma k} A_{\sigma j}+A_{\sigma j}^{\prime} X_{1 \sigma k}^{\prime} & P_{j k}-X_{1 \sigma k}+A_{\sigma j}^{\prime} X_{2 \sigma k}^{\prime} \\
P_{j k}-X_{1 \sigma k}^{\prime}+X_{2 \sigma k} A_{\sigma j} & -X_{2 \sigma k}-X_{2 \sigma k}^{\prime}
\end{array}\right] \\
& \times\left[\begin{array}{c}
I_{n} \\
A(\sigma, \alpha)
\end{array}\right] x \text {. }
\end{aligned}
$$

Now, define $P(\alpha)=\left(\alpha_{1} \alpha_{1} P_{11}+\alpha_{1} \alpha_{2} P_{12}+\ldots+\alpha_{r} \alpha_{r} P_{r r}\right), X_{1}(\sigma, \alpha)=$ $\left(\alpha_{1} X_{1 \sigma 1}+\alpha_{2} X_{1 \sigma 2}+\ldots+\alpha_{r} X_{1 \sigma r}\right), X_{2}(\sigma, \alpha)=\left(\alpha_{1} X_{2 \sigma 1}+\alpha_{2} X_{2 \sigma 2}+\ldots+\right.$ 
$\alpha_{r} X_{2 \sigma r}$ ). Then, from (2) and (A3), one has:

$0>x^{\prime}\left[\begin{array}{c}I_{n} \\ A(\sigma, \alpha)\end{array}\right]^{\prime}\left[\begin{array}{c}X_{1}(\sigma, \alpha) A(\sigma, \alpha)+A^{\prime}(\sigma, \alpha) X_{1}^{\prime}(\sigma, \alpha) \\ P(\alpha)-X_{1}^{\prime}(\sigma, \alpha)+X_{2}(\sigma, \alpha) A(\sigma, \alpha)\end{array}\right.$

$\left.\begin{array}{c}P(\alpha)-X_{1}(\sigma, \alpha)+A^{\prime}(\sigma, \alpha) X_{2}^{\prime}(\sigma, \alpha) \\ -X_{2}(\sigma, \alpha)-X_{2}^{\prime}(\sigma, \alpha)\end{array}\right]\left[\begin{array}{c}I_{n} \\ A(\sigma, \alpha)\end{array}\right] x$

$=x^{\prime}\left[\begin{array}{c}I_{n} \\ A^{\prime}(\sigma, \alpha)\end{array}\right]^{\prime}\left\{\left[\begin{array}{cc}0 & P(\alpha) \\ P(\alpha) & 0\end{array}\right]+\left[\begin{array}{l}X_{1}(\sigma, \alpha) \\ X_{2}(\sigma, \alpha)\end{array}\right]\left[\begin{array}{ll}A(\sigma, \alpha) & -I_{n}\end{array}\right]\right.$

$$
\begin{aligned}
& \left.+\left[\begin{array}{c}
A^{\prime}(\sigma, \alpha) \\
-I_{n}
\end{array}\right]\left[\begin{array}{ll}
X_{1}^{\prime}(\sigma, \alpha) & X_{2}^{\prime}(\sigma, \alpha)
\end{array}\right]\right\}\left[\begin{array}{c}
I_{n} \\
A(\sigma, \alpha)
\end{array}\right] x \\
& =x^{\prime}\left[\begin{array}{ll}
I_{n} & A^{\prime}(\sigma, \alpha)
\end{array}\right]\left[\begin{array}{cc}
0 & P(\alpha) \\
P(\alpha) & 0
\end{array}\right]\left[\begin{array}{c}
I_{n} \\
A(\sigma, \alpha)
\end{array}\right] x .
\end{aligned}
$$

Considering a Lyapunov function candidate $V(x)=x^{\prime} P(\alpha) x$, note that from (2), $V(x)>0$ for $x \neq 0$ and from (1), supposing that $u(t)=0$ and $w(t)=0$ for $t \geq 0$, and assuming constant output matrices $C_{2 j}=C$, for all $j \in \mathbb{K}_{r}$, and (A4) it follows that $\dot{V}(x)=\dot{x}(t)^{\prime} P(\alpha) x(t)+x(t)^{\prime} P(\alpha) \dot{x}(t)<0$ for $x \neq 0$. The proof is concluded. 